\title{
Effect of impurities in the description of surface nanobubbles: Role of nonidealities in the surface layer
}

\author{
Siddhartha Das* \\ Physics of Fluids, Faculty of Science and Technology, University of Twente, P.O. Box 217, NL-7500 AE Enschede, The Netherlands
}

(Received 23 December 2010; revised manuscript received 31 March 2011; published 20 June 2011)

\begin{abstract}
In a recent study [S. Das, J. H. Snoeijer, and D. Lohse, Phys. Rev. E 82, 056310 (2010)], we provided quantitative demonstration of the conjecture [W. A. Ducker, Langmuir 25, 8907 (2009)] that the presence of impurities at the surface layer (or the air-water interface) of surface nanobubbles can substantially lower the gas-side contact angle and the Laplace pressure of the nanobubbles. Through an analytical model for any general air-water interface without nonideality effects, we showed that a large concentration of soluble impurities at the air-water interface of the nanobubbles ensures significantly small contact angles (matching well with the experimental results) and Laplace pressure (though large enough to forbid stability). In this paper this general model is extended to incorporate the effect of nonidealities at the air-water interface in impurity-induced alteration of surface nanobubble properties. Such nonideality effects arise from finite enthalpy or entropy of mixing or finite ionic interactions of the impurity molecules at the nanobubble air-water interface and ensure significant lowering of the nanobubble contact angle and Laplace pressure even at relatively small impurity coverage. In fact for impurity molecules that show enhanced tendency to get adsorbed at the nanobubble air-water interface from the bulk phase, impurity-induced lowering of the nanobubble contact angle is witnessed for extremely small bulk concentration. Surface nanobubble experiments being typically performed in an ultraclean environment, the bulk concentration of impurities is inevitably very small, and in this light the present calculations can be viewed as a satisfactory explanation of the conjecture that impurities, even in trace concentration, have significant impact on surface nanobubbles.
\end{abstract}

DOI: 10.1103/PhysRevE.83.066315

PACS number(s): 47.55.db, 68.08.-p, 68.03.-g

\section{INTRODUCTION}

"Surface nanobubbles" refer to small spherical caplike air (vapor) bubbles formed at the solid-liquid interface of submerged hydrophobic substrates. Over the past decade, there has been large number of investigations studying the formation, the properties, and the applications of these bubbles [1-16]. Most of these studies are nicely summarized in a couple of recent review articles $[17,18]$. Great scientific interest in surface nanobubbles stems from the fact that unlike macroscopic or even microscopic bubbles, many of their reported properties (the properties of surface nanobubbles that are typically of concern are their gas-side contact angle $\theta$ and radius $R_{b}$; see Fig. 1 of [19] as well as Fig. 1 provided here) cannot be trivially explained from surface tension values for the media involved. For example, for most of the substrates, the surface nanobubbles exhibit a gas-side contact angle much smaller than the corresponding Young's angle $\theta_{\mathrm{Y}}$ [20]. Surface nanobubbles formed on octadecyltrichlorosilane (OTS)-silicon-water interface, for example, are found to have a gas-side contact angle of $\sim 20^{\circ}[2,7,20]$, whereas the corresponding $\theta_{\mathrm{Y}}$ [applying Young's equation $\cos \theta_{Y}=\left(\sigma_{s l}-\sigma_{s g}\right) / \sigma_{\mathrm{lg}}$, with surface tension values $\sigma_{\mathrm{lg}}=$ $0.072 \mathrm{~N} / \mathrm{m}$ and $\left.\sigma_{s l}-\sigma_{s g}=0.025 \mathrm{~N} / \mathrm{m}\right]$ is $\sim 70^{\circ}$. An even more contentious issue concerning surface nanobubbles is their extremely great stability. Bubbles with radii $\sim 100 \mathrm{~nm}$ will experience a Laplace pressure $(\Delta p) \sim \mathrm{MPa}$ (e.g., for OTS-silicon-water interface, $\Delta p=\frac{2 \sigma_{\mathrm{lg}}}{R_{b}}=1.44 \mathrm{MPa}$, for $R_{b}=$

*s.das@utwente.nl; siddhartha_iit@yahoo.com
$100 \mathrm{~nm}$ ) suggesting that the nanobubble will dissolve almost instantly after its formation. However, experimental studies report that the nanobubbles can remain stable for several days when left undisturbed $[12,15]$. Various explanations have been proposed to resolve these two issues [20-26], though a unanimous understanding is yet to be reached.

In a recent study Borkent et al. [8], through concrete experimental evidence, suggested that the anomalously small nanobubble (gas-side) contact angle might be attributed to the unavoidable presence of trace amounts of impurities in the experimental setup. Ducker [20] had earlier conjectured that the presence of such impurities at the nanobubble air-water interface might act as a shield to the outflux of gases (making the bubbles more stable) and also reduce the contact angle and Laplace pressure by lowering the surface tension $\sigma_{\mathrm{lg}}$. However, with surface adsorption of soluble impurities, the air-water equilibrium surface tension can never drop below 0.025$0.03 \mathrm{~N} / \mathrm{m}$. This value is not small enough to ensure nanobubble superstability. Thus, Ducker [20] hypothesized that in addition to the soluble impurities, certain insoluble impurity molecules might get stuck on the bubble, forcing the air-water surface tension to a value much lower than $0.025 \mathrm{~N} / \mathrm{m}$, thereby making the surface nanobubbles extremely stable.

To the best of our knowledge, however, there was no mathematical model describing the effect of impurities on surface nanobubbles. To address this issue we recently provided a general mathematical framework that pinpoints the effect of impurities (soluble) on the lowering of the surface tension of any general air-water interface and used this model to quantify the effect of impurities in the alteration of surface nanobubble properties [19]. Assuming ideal interactions at the impurity-covered nanobubble air-water interface (we may 


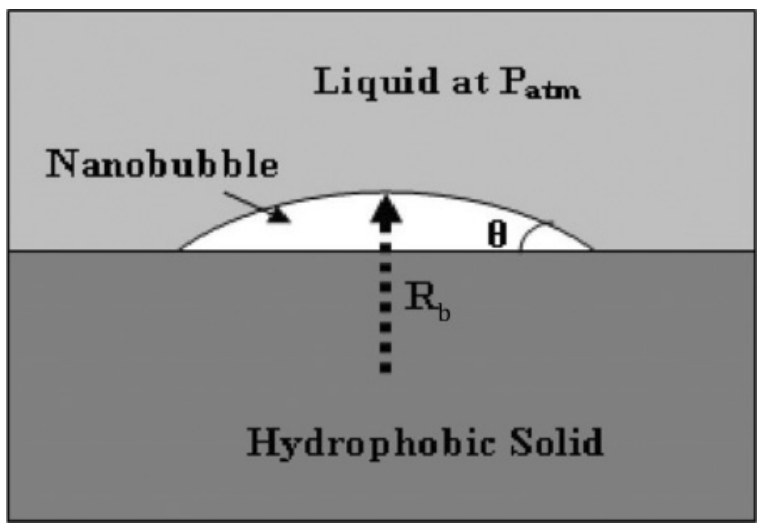

FIG. 1 . Schematic of a nanobubble. $\theta$ : gas-side contact angle; $R_{b}$ : radius of the bubble.

interchangeably call the nanobubble air-water interface the nanobubble surface layer), the model predicted significant reductions in gas-side contact angles (to values that matched experimental results $[2,7,20]$ ) and Laplace pressure (the Laplace pressure, though, is not lowered significantly to justify nanobubble superstability) for substantial impurity surface coverage [19].

In the present paper this model is thoroughly elaborated to ensure that the effect of nonidealities in the nanobubble air-water interface are appropriately accounted for in the estimation of impurity-induced alteration of surface nanobubble properties. The nonideality effects arise from the enthalpic, entropic, and ionic interactions between the surface-adsorbed impurity molecules, and become particularly relevant at surface coverage values at which impurity effects are found to become important for an ideal nanobubble air-water interface [19]. The nonideality based model presented in this paper is thus much broader and more general in scope than our previous simplified ideality based model [19]. The present model is first validated by making it reproduce the reported experimental results of surfactant-induced lowering of surface tension of simple air-water interfaces. The model is next employed to study the effect of impurities on surface nanobubbles. The most important result obtained from the present calculations, in context of surface nanobubbles, is that the nonideality effects ensure that even relatively weak impurity coverage (much smaller than that corresponding to the ideality-based model [19]) can lead to significant alteration of gas-side contact angle and Laplace pressure of surface nanobubbles. Though the Laplace pressure is still high enough to forbid stability (and hence the nanobubble superstability puzzle is still unsolved), the gas-side contact angle shows values close to experimental results $[2,7,20]$ even for substantially reduced impurity coverage. In fact, for impurities that show a greater propensity to get adsorbed at the nanobubble air-water interface from the bulk solution, our model demonstrates that the nanobubble contact angle gets lowered (to experimental values) even for extremely small values of the bulk concentration (of the order of only a few micromoles or even less). As experiments on surface nanobubbles are invariably carried out in an extremely clean environment, one can expect the presence of only trace amount of impurities in the system.
In this light, the present nonideality-based model not only provides a more acceptable foundation (in comparison to our previous ideality-based model [19]) for delineating the role of impurities on surface nanobubbles [20], but also provides a satisfactory explanation on how impurities, even in trace amounts, can lead to anomalously small nanobubble contact angles.

\section{THEORY}

The presence of soluble impurities at the air-water interface can at most cancel the component of the air-water surface tension that is contributed by the hydrogen bond (HB)induced interaction forces, whereas the contribution due to the dispersion forces is always present (for more details see [19]). Consequently, the equilibrium air-water surface tension never drops below $0.03-0.025 \mathrm{~N} / \mathrm{m}$. The model, which will follow, is based on a general equilibrium description of surfactant adsorption at the air-water interface [27-29] and is valid under the assumption that the adsorption time scale is much smaller than the characteristic time scale that can be associated with the surface nanobubbles [19]. The equilibrium picture starts with the condition that the chemical potential at the air-water interface is identical to that in the bulk solution for any kind of impurity as well as the solvent molecules. Thus one may write (with $m=i$ for the $i$ th type of impurity and $m=0$ for the solvent) [19,27-29]:

$$
\mu_{m}^{0 s}-\omega_{m} \sigma_{\mathrm{lg}}^{\prime}+R T \ln \left(f_{m}^{s} x_{m}^{s}\right)=\mu_{m}^{0 b}+R T \ln \left(f_{m}^{b} x_{m}^{b}\right) .
$$

In Eq. (1), $\mu_{m}^{0 s}$ and $\mu_{m}^{0 b}$ are the standard state chemical potentials at the air-water interface and the bulk, $f_{m}^{s}$ and $f_{m}^{b}$ are the activity coefficients at the air-water interface and the bulk, and $x_{m}^{s}$ and $x_{m}^{b}$ are the mole fraction at the air-water interface and the bulk (superscript " $s$ " refers to the air-water interface or the surface layer and superscript " $b$ " refers to the bulk solution). Also $\omega_{m}$ is the partial molar area of the moieties (impurity or water), $\sigma_{\mathrm{lg}}^{\prime}$ is the modified value of the surface tension in the presence of impurities, $R$ is the universal gas constant, and $T$ is the absolute temperature.

Equation (1) can be further simplified with the equations for standard states, expressed as (conditions under which the equations for standard states are obtained can be found in Refs. [19,29]; for the impurity, $m=i$ and for the solvent, $m=0$ )

$$
\mu_{0}^{0 s}-\omega_{0} \sigma_{\lg }=\mu_{0}^{0 b},
$$

and

$$
\mu_{i}^{0 b}-\mu_{i}^{0 s}=-\omega_{i} \sigma_{\mathrm{lg}}+R T \ln \left(K_{i}\right)+R T \ln \left(\frac{f_{(0) i}^{s}}{f_{(0) i}^{b}}\right),
$$

where $\sigma_{\mathrm{lg}}$ is the surface tension without impurities, $K_{i}=$ $\left(x_{i}^{s} / x_{i}^{b}\right)_{x_{i}^{b} \rightarrow 0}, f_{(0) i}^{b}=\left(f_{i}^{b}\right)_{x_{i}^{b} \rightarrow 0}$ (bulk activity coefficient of the impurity at infinite dilution), and $f_{(0) i}^{s}=\left(f_{i}^{s}\right)_{x_{i}^{b} \rightarrow 0}$ (surface activity coefficient of the impurity at infinite dilution).

Combining Eqs. (1)-(3), one can obtain the equations of states (in terms of the surface pressure $\Pi$, where $\Pi=\sigma_{\mathrm{lg}}-\sigma_{\mathrm{lg}}^{\prime}$ ) 
for the solvent and the impurity molecules as

$$
\begin{aligned}
\ln \left(\frac{f_{0}^{s} x_{0}^{s}}{f_{0}^{b} x_{0}^{b}}\right) & =-\frac{\omega_{0}\left(\sigma_{\lg }-\sigma_{\mathrm{lg}}^{\prime}\right)}{R T} \\
& =-\frac{\omega_{0} \Pi}{R T} \text { (equation of state for the solvent), }
\end{aligned}
$$

and

$$
\begin{aligned}
& \ln \left(\frac{f_{i}^{s} x_{i}^{s} / f_{(0) i}^{s}}{K_{i} f_{i}^{b} x_{i}^{b} / f_{(0) i}^{b}}\right) \\
& =-\frac{\omega_{i}\left(\sigma_{\mathrm{lg}}-\sigma_{\mathrm{lg}}^{\prime}\right)}{R T} \\
& \left.=-\frac{\omega_{i} \Pi}{R T} \text { (equation of state for the impurity } i\right) .
\end{aligned}
$$

One can further simplify the above equations by assuming ideality of the bulk solution (this implies that in the present study, nonideality effects are considered only at the air-water interface). Also, the bulk concentration of the impurities is assumed to be relatively small. These conditions finally lead to

$$
f_{0}^{b}=1, \quad f_{(0) i}^{b}=1, \quad f_{i}^{b}=1, \quad x_{0}^{b} \rightarrow 1 .
$$

Using Eq. (6) one can simplify Eqs. (4) and (5) as:

$$
\ln \left(f_{0}^{s} x_{0}^{s}\right)=-\frac{\omega_{0} \Pi}{R T},
$$

and

$$
\ln \left(\frac{f_{i}^{s} x_{i}^{s}}{K_{i} f_{(0) i}^{s} x_{i}^{b}}\right)=-\frac{\omega_{i} \Pi}{R T} .
$$

It is further assumed that the values of partial molar areas of the impurities are identical, i.e., $\omega_{1}=\omega_{2}=\cdots .=\omega_{n}$. Under this condition, the mole fraction of the impurities (at the air-water interface) is identical to their respective fraction coverage (at the air-water interface) $\beta_{i}$ (where $\beta_{i}=\omega_{i} \Gamma_{i}$, with $\Gamma_{i}$ being the adsorption of the impurity of type $i$ ) [27]. Consequently, Eqs. (7) and (8) can be written as

$$
\Pi=-\frac{R T}{\omega_{0}}\left[\ln \left(1-\sum_{i \geqslant 1} \beta_{i}\right)+\ln \left(f_{0}^{s}\right)\right],
$$

and

$$
\beta_{i}=\frac{K_{i} x_{i}^{b} f_{(0) i}^{s}}{f_{i}^{s}} \exp \left(-\frac{\omega_{i} \Pi}{R T}\right) .
$$

One can finally use Eqs. (9) and (10) to obtain the surface pressure (and consequently the nanobubble parameters $\theta$ and $\Delta p$ ) for the cases of nonionic and ionic impurities.

\section{A. Nonionic impurities}

It is clear from Eq. (9) that to obtain the surface pressure one needs to first calculate the activity coefficient $f_{0}^{s}$, representing the effect of nonideal interactions at the air-water interface. It is in this context that the present model drastically differs from the one proposed in our previous study [19], where ideality assumptions meant $f_{0}^{s}=1\left[\right.$ or $\left.\ln \left(f_{0}^{s}\right)=0\right]$. For the case of nonionic impurities, nonideality effects result from finite enthalpy and entropy of mixing of impurity molecules at the air-water interface. These two effects being additive in the Gibbs free energy, one may write [27]

$$
f_{0}^{s}=f_{0}^{s, \text { enth }} f_{0}^{s, \text { entr }},
$$

which gives

$$
\ln \left(f_{0}^{s}\right)=\ln \left(f_{0}^{s, \text { enth }}\right)+\ln \left(f_{0}^{s, \text { entr }}\right) .
$$

In Eqs. (11) and (12) $f_{0}^{s, \text { entr }}$ and $f_{0}^{s, \text { enth }}$ are the respective contributions of the entropic and enthaplic nonideality effects to the overall activity coefficient $f_{0}^{s}$.

As in $[27,29,30]$, one can express $f_{0}^{s, \text { entr }}$ as:

$$
\ln \left(f_{0}^{s, \text { entr }}\right)=n_{0} \sum_{i} \frac{\beta_{i}}{n_{i}}-1,
$$

where $n_{k}=\omega_{k} / \omega_{0}$.

For a case where there are two types of impurities, Eq. (13) reduces to

$$
\ln \left(f_{0}^{s, \text { entr }}\right)=-\beta_{1}\left(1-\frac{1}{n_{1}}\right)-\beta_{2}\left(1-\frac{1}{n_{2}}\right) .
$$

$f_{0}^{s, \text { enth }}$ depends on the energies of interaction between molecules of impurities of similar and/or different types present at the air-water interface. Using the regular solution theory [31-33], one can write

$$
f_{0}^{s, \text { enth }}=\frac{1}{R T} \sum_{i} \sum_{j}\left(A_{i 0}^{s}-\frac{1}{2} A_{i j}^{s}\right) \beta_{i} \beta_{j},
$$

where $A_{i j}^{s}$ can be expressed in terms of interaction energies $\left(U_{i i}^{s}, U_{j j}^{s}\right.$, and $U_{i j}^{s}$ ) between the moieties (similar and/or different) present at the air-water interface as

$$
A_{i j}^{s}=U_{i i}^{s}+U_{j j}^{s}-2 U_{i j}^{s} .
$$

Importantly, these different interaction energies are independent of the fraction coverage of the impurities. Consequently, Eq. (15) can be written in a simplified form, which, for the case where there are two different types of impurities at the air-water interface, can be expressed as [27,29]

$$
\ln \left(f_{0}^{s, \text { enth }}\right)=a_{11} \beta_{1}^{2}+a_{22} \beta_{1}^{2}+a_{12} \beta_{1} \beta_{2},
$$

where $a_{11}, a_{22}$, and $a_{12}$ are the constants (independent of the fraction coverage of impurities) that depend on the interaction energies between the species. These constants can be evaluated with known values of interaction potentials between the impurity molecules. In the present calculation the dominant form of interaction between the uncharged impurity molecules is assumed to be dipole-dipole interactions (similar to the study of Karakashev and Manev [34]), although it may be noted that calculations are equally possible for other forms of interactions (such as van der Waals interactions or Lennard-Jones interactions) as well. Also by considering only dipole-dipole interactions, we neglect the possible orientationinduced interactions [e.g., interactions of adjacently located hydrophobic heads of oriented surfactant molecules [35] at the air-water interface]. Karakashev and Manev [34] considered nonideality effects to be solely due to the enthalpic 
interactions. With similar considerations, the equation of state [Eq. (9)] gets reduced to [using Eq. (17)]

$$
\Pi=\Pi_{\text {ideal }}-\frac{R T}{\omega_{0}}\left(a_{11} \beta_{1}^{2}+a_{22} \beta_{1}^{2}+a_{12} \beta_{1} \beta_{2}\right),
$$

where $\Pi_{\text {ideal }}=-\frac{R T}{\omega_{0}}\left[\ln \left(1-\beta_{1}-\beta_{2}\right)\right]$ is the surface pressure without nonideal effects. reads

The equivalent expression in Karakashev and Manev [34]

$$
\Pi=\Pi_{\text {ideal }}-\left(b_{11} \Gamma_{1}^{2}+b_{22} \Gamma_{2}^{2}+b_{12} \Gamma_{1} \Gamma_{2}\right),
$$

where $\Gamma_{1}=\beta_{1} / \omega_{1}$ and $\Gamma_{2}=\beta_{2} / \omega_{2}$ are the adsorption of the impurities at the air-water interface.

Comparing Eqs. (18) and (19), one obtains

$$
\begin{aligned}
& a_{11}=\frac{\omega_{0}}{R T \omega_{1}^{2}} b_{11}, \quad a_{22}=\frac{\omega_{0}}{R T \omega_{2}^{2}} b_{22}, \\
& a_{12}=\frac{\omega_{0}}{R T \omega_{1} \omega_{2}} b_{12} .
\end{aligned}
$$

Using [34], the parameter $b_{i j}$ (having units of $\mathrm{J} \mathrm{m}^{2} / \mathrm{mol}^{2}$ ) can be expressed in terms of the dipole-dipole energy of interaction between species $i$ and $j\left[U_{i j}^{d p-d p}(r)\right]$ as

$$
b_{i j}=-\pi N_{A}^{2} \int_{d_{i j}}^{\infty} U_{i j}^{d p-d p}(r) r d r,
$$

where, $N_{A}$ is Avogadro's number, $d_{i j}$ is the minimum separation distance between the molecules of impurities $i$ and $j$, and $U_{i j}^{d p-d p}(r)$ can be expressed as a function of the separation distance $r$ as

$$
U_{i j}^{d p-d p}(r)=-\frac{1}{\left(4 \pi \varepsilon_{0} \varepsilon_{r}\right)^{2}} \frac{2 p_{i}^{2} p_{j}^{2}}{k_{B} T r^{6}},
$$

with $p_{i}$ and $p_{j}$ being the dipole moments of the molecules of type $i$ and $j$.

Using Eq. (22) in Eq. (21), one will get

$$
\begin{gathered}
b_{11}=-\frac{1}{\left(4 \pi \varepsilon_{0} \varepsilon_{r}\right)^{2}} \frac{2 \pi N_{A}^{2} p_{1}^{4}}{5 k_{B} T d_{11}^{4}}, \\
b_{22}=-\frac{1}{\left(4 \pi \varepsilon_{0} \varepsilon_{r}\right)^{2}} \frac{2 \pi N_{A}^{2} p_{2}^{4}}{5 k_{B} T d_{22}^{4}}, \\
b_{12}=-\frac{1}{\left(4 \pi \varepsilon_{0} \varepsilon_{r}\right)^{2}} \frac{2 \pi N_{A}^{2} p_{1}^{2} p_{2}^{2}}{5 k_{B} T d_{12}^{4}} .
\end{gathered}
$$

Thus using Eqs. (20) and (23), one may obtain the expressions for $a_{11}, a_{22}$, and $a_{12}$. Subsequently using Eqs. (9), (12), (14), and (17), the equation for the surface pressure for nonionic impurities with nonideality considerations can be expressed as (for two types of impurities at the air-water interface)

$$
\begin{aligned}
\Pi= & -\frac{R T}{\omega_{0}}\left[\ln \left(1-\beta_{1}-\beta_{2}\right)\right] \\
& +\frac{R T}{\omega_{0}}\left[\beta_{1}\left(1-\frac{\omega_{0}}{\omega_{1}}\right)+\beta_{2}\left(1-\frac{\omega_{0}}{\omega_{2}}\right)\right] \\
& +\frac{1}{\left(4 \pi \varepsilon_{0} \varepsilon_{r}\right)^{2}} \frac{2 \pi N_{A}^{2}}{5 k_{B} T}\left[\frac{p_{1}^{4}}{d_{11}^{4}} \frac{\beta_{1}^{2}}{\omega_{1}^{2}}+\frac{p_{2}^{4}}{d_{22}^{4}} \frac{\beta_{2}^{2}}{\omega_{2}^{2}}+\frac{p_{1}^{2} p_{2}^{2}}{d_{12}^{4}} \frac{\beta_{1} \beta_{2}}{\omega_{1} \omega_{2}}\right] .
\end{aligned}
$$

Under the assumption that the size of the impurity molecules is always larger than the water molecule (i.e., $\omega_{\mathrm{i}} \geqslant$ $\omega_{0}$ ), both the entropic [second term on the right-hand side of Eq. (24)] as well as the enthalpic [third term on the right-hand side of Eq. (24)] nonidealities are found to increase the surface pressure. This establishes that nonideality consideration (for nonionic impurities) ensures a more pronounced effect of the impurities in lowering the surface tension of an air-water interface.

\section{B. Ionic impurities}

For ionic impurities, even the case of ideal air-water interface involves an additional surface pressure contribution, over and above the contribution due to surface coverage of impurities, expressed as [19,36,37]

$$
\Delta \Pi_{\text {ionic }} \approx 2 R T \sum_{i}\left|z_{s, i}\right| \Gamma_{s, i}=2 R T \sum_{i} \frac{\left|z_{s, i}\right| \beta_{i}}{\omega_{i}} .
$$

Equation (25) is derived under the assumption that there are $i$ types of ionic impurities (with valence $z_{s}, i$ ) adsorbed at the air-water interface with all the ions acting as potential determining ions [19,36,37].

Consequently, the ideal case surface pressure is expressed as (for one kind of impurity, which is ionic, at the air-water interface) $[19,27]$

$$
\begin{aligned}
\Pi_{\text {ionic }} & =\Pi_{\text {ideal }}+\Delta \Pi_{\text {ionic }} \\
& =-\frac{R T}{\omega_{0}}\left[\ln \left(1-\beta_{1}\right)\right]+\frac{2 R T\left|z_{s}\right| \beta_{1}}{\omega_{1}} .
\end{aligned}
$$

The nonideality description for the ionic impurities will necessarily involve additional ionic interactions between the impurity molecules. This will alter the enthalpic component of nonideality (as it depends on the nature of interactions between the impurity molecules), but the entropic component will remain unchanged. Consequently the interaction parameter $b_{i j}$ [appearing in Eq. (21)] can be expressed as [35,38] (neglecting the contribution due to the orientation of the molecules $[35,38]$ )

$b_{i j}=-\pi N_{A}^{2} \int_{d_{i j}}^{\infty} U_{i j}^{d p-d p}(r) r d r-\pi N_{A}^{2} \int_{d_{i j}}^{\infty} U_{i j}^{D H}(r) r d r$.

Equation (27) assumes that the ionic impurities adsorbed at the air-water interface form an electric double layer (EDL), and accordingly the ionic interactions between species $i$ and $j$ (with valences $z_{i}$ and $z_{j}$ ) are described by the Debye-Hückel interaction $U_{i j}^{\mathrm{DH}}(r)$ expressed as $[35,38]$

$$
U_{i j}^{\mathrm{DH}}(r)=-\frac{z_{i} z_{j} e^{2}}{4 \pi \varepsilon_{0} \varepsilon_{r}} \exp (-\kappa r),
$$

where $\kappa$ is the inverse of the Debye layer thickness.

Using Eq. (28) in Eq. (27), one can get (for monovalent impurities)

$$
b_{i j}=-\frac{1}{\left(4 \pi \varepsilon_{0} \varepsilon_{r}\right)^{2}} \frac{2 \pi N_{A}^{2} p_{i}^{2} p_{j}^{2}}{5 k_{B} T d_{i j}^{4}}-\frac{F^{2}}{4 \varepsilon_{0} \varepsilon_{r} \kappa} \exp \left(-\kappa d_{i j}\right),
$$

where $F$ is the Faraday constant.

For a relatively small concentration of ionic impurities the Debye layer thickness is large (or $\kappa$ is relatively small); 
hence $\kappa d_{i j} \ll 1$ (since $d_{i j}$ is of the order of ionic diameter) or $\exp \left(-\kappa d_{i j}\right) \approx 1$. Consequently, one can simplify Eq. (29) as

$$
b_{i j}=-\frac{1}{\left(4 \pi \varepsilon_{0} \varepsilon_{r}\right)^{2}} \frac{2 \pi N_{A}^{2} p_{i}^{2} p_{j}^{2}}{5 k_{B} T d_{i j}^{4}}-\frac{F^{2}}{4 \varepsilon_{0} \varepsilon_{r} \kappa} .
$$

Subsequently, using Eqs. (9), (12), (14), (17), (20), and (30), the surface pressure for ionic impurities with nonideality effects can be expressed as (for two types of impurities, both ionic, at the air-water interface, with all the impurity ions acting as surface-active or potential-determining ions)

$$
\begin{aligned}
\Pi= & -\frac{R T}{\omega_{0}}\left[\ln \left(1-\beta_{1}-\beta_{2}\right)\right]+2 R T\left[\frac{\left|z_{s 1}\right| \beta_{1}}{\omega_{1}}+\frac{\left|z_{s 1}\right| \beta_{2}}{\omega_{2}}\right] \\
& +\frac{R T}{\omega_{0}}\left[\beta_{1}\left(1-\frac{\omega_{0}}{\omega_{1}}\right)-\beta_{2}\left(1-\frac{\omega_{0}}{\omega_{2}}\right)\right] \\
& +\frac{1}{\left(4 \pi \varepsilon_{0} \varepsilon_{r}\right)^{2}} \frac{2 \pi N_{A}^{2}}{5 k_{B} T}\left[\frac{p_{1}^{4}}{d_{11}^{4}} \frac{\beta_{1}^{2}}{\omega_{1}^{2}}+\frac{p_{2}^{4}}{d_{22}^{4}} \frac{\beta_{2}^{2}}{\omega_{2}^{2}}+\frac{p_{1}^{2} p_{2}^{2}}{d_{12}^{4}} \frac{\beta_{1} \beta_{2}}{\omega_{1} \omega_{2}}\right] \\
& +\frac{F^{2}}{4 \varepsilon_{0} \varepsilon_{r} \kappa}\left[\frac{\beta_{1}^{2}}{\omega_{1}^{2}}+\frac{\beta_{2}^{2}}{\omega_{2}^{2}}+\frac{\beta_{1} \beta_{2}}{\omega_{1} \omega_{2}}\right] .
\end{aligned}
$$

Equation (31) shows that the surface pressure for ionic impurities depends on the inverse Debye length $\kappa$. As a result, unlike the case for the nonionic impurities, the surface pressure will no longer be a single-valued function of fractional surface coverage of impurities $\beta$ but will also depend on bulk concentration of impurities (as $\kappa$ depends on bulk concentration). Thus to obtain the surface pressure, one needs to solve Eq. (31) iteratively along with Eq. (10), which relates the surface pressure with the bulk concentration. Under the condition of weak bulk ionic concentration, (i.e., $x_{0}^{b} \gg x_{i}^{b}$, or $x_{i}^{b} \approx$ $\frac{\text { number of moles of impurities }}{\text { number of moles of water }}=\frac{\text { number density of impurities }}{\text { number density of water }}$ ), Eq. (10) can be rearranged to interconnect the surface pressure with bulk number density $\xi_{i}$ (in units of $1 / \mathrm{m}^{3}$ ) of ionic impurity of type $i$ as (under the condition $f_{(0) i}^{s} \rightarrow 1$ )

$$
\xi_{i}=\frac{1000}{0.018} \frac{N_{A} f_{i}^{s} \beta_{i}}{K_{i}} \exp \left(\frac{\omega_{i} \Pi}{R T}\right) .
$$

Equation (32) considers the density of water as 1000 $\mathrm{kg} / \mathrm{m}^{3}$ and the one gram-molecular weight weight of water as $0.018 \mathrm{~kg}$, implying that the number density of water is $1000 N_{\mathrm{A}} / 0.0181 / \mathrm{m}^{3}$. To use Eqs. (31) and (32) simultaneously, one needs to know the parameters $K_{i}$ and $f_{i}^{s}$ a priori. The activity coefficients $f_{i}^{s}$ of the individual impurities can be expressed as functions of the interaction parameters $a_{i j}$ and the surface coverage $\beta_{i}$ as (for a case with two types of ionic impurities at the air-water interface) [27]

$$
\begin{aligned}
& f_{1}^{s}=\exp \left(a_{11} \beta_{1}^{2}+a_{22} \beta_{1}^{2}+a_{12} \beta_{1} \beta_{2}-2 a_{11} \beta_{1}-2 a_{12} \beta_{2}\right), \\
& f_{2}^{s}=\exp \left(a_{11} \beta_{1}^{2}+a_{22} \beta_{1}^{2}+a_{12} \beta_{1} \beta_{2}-2 a_{12} \beta_{1}-2 a_{22} \beta_{2}\right) .
\end{aligned}
$$

In Eq. (33) all $a_{i j}$ are expressed using Eqs. (20) and (30). Consequently, all $f_{i}^{s}$ also become functions of $\kappa$, which can be connected to the bulk ionic number densities of the impurities $\left(\xi_{i}\right)$ as

$$
\kappa=\sqrt{\frac{2 e^{2}\left(z_{1}^{2} \xi_{1}+z_{2}^{2} \xi_{2}\right)}{\varepsilon_{0} \varepsilon_{r} k_{B} T}} .
$$

Equation (34) assumes that the two types of impurities come from two different salts, each of which has symmetric (z:z) valences; thus in the bulk the total ionic number density is $2\left(\xi_{1}+\xi_{2}\right)$. Accordingly, for a given surface coverage of the two types of impurities (namely, $\beta_{1}$ and $\beta_{2}$ ), one has four unknowns (namely $\xi_{1}, \xi_{2}, \Pi$, and $\kappa$ ) and four equations [i.e., Eqs. (31), (34), and (32) (representing two equations)]. Equivalently, one may also consider the known variables as the bulk number densities $\xi_{1}$ and $\xi_{2}$, in which case there are again four unknowns, namely $\beta_{1}, \beta_{2}, \Pi$, and $\kappa$ and the same four equations.

In case there is only one kind of ionic impurity at the airwater interface and its neutralizing ion (forming a z:z salt) remains outside the air-water interface, one can get a much simplified picture, which can be addressed analytically. This case is discussed below, under the condition that there is a known value of the fraction coverage of ionic impurities $\beta_{1}$ at the air-water interface.

At a given value of $\beta_{1}$, one can rewrite Eq. (31) as

$$
\Pi=C_{1}+\frac{C_{2}}{\kappa},
$$

where

$$
\begin{aligned}
C_{1}= & -\frac{R T}{\omega_{0}}\left[\ln \left(1-\beta_{1}\right)\right]+\frac{2 R T\left|z_{s 1}\right| \beta_{1}}{\omega_{1}} \\
& +\frac{R T}{\omega_{0}}\left[\beta_{1}\left(1-\frac{\omega_{0}}{\omega_{1}}\right)\right]+\frac{1}{\left(4 \pi \varepsilon_{0} \varepsilon_{r}\right)^{2}} \frac{2 \pi N_{A}^{2}}{5 k_{B} T}\left[\frac{p_{1}^{4}}{d_{11}^{4}} \frac{\beta_{1}^{2}}{\omega_{1}^{2}}\right]
\end{aligned}
$$

and

$$
C_{2}=\frac{F^{2}}{4 \varepsilon_{0} \varepsilon_{r}} \frac{\beta_{1}^{2}}{\omega_{1}^{2}} .
$$

From Eqs. (32) and (33), one gets

$$
\xi_{1}=C_{3} \exp \left(\frac{C_{4}}{\kappa}+C_{5} \Pi\right),
$$

where

$$
\begin{aligned}
C_{3}= & \frac{1000}{0.018} \frac{N_{A} \beta_{1}}{K_{1}} \\
& \times \exp \left\{-\frac{\omega_{0}}{R T \omega_{1}^{2}}\left[\frac{1}{\left(4 \pi \varepsilon_{0} \varepsilon_{r}\right)^{2}} \frac{2 \pi N_{A}^{2} p_{1}^{4}}{d_{11}^{4}}\right]\left(\beta_{1}^{2}-2 \beta_{1}\right)\right\} \\
C_{4}= & -\frac{F^{2} \omega_{0}}{4 \pi \varepsilon_{0} \varepsilon_{r} R T \omega_{1}^{2}}\left(\beta_{1}^{2}-2 \beta_{1}\right)
\end{aligned}
$$

and

$$
C_{5}=\frac{\omega_{1}}{R T} .
$$

Also using Eq. (35) in Eq. (38), one gets

$$
\xi_{1}=C_{6} \exp \left(\frac{C_{7}}{\kappa}\right)
$$

where

$$
C_{6}=C_{3} \exp \left(C_{1} C_{5}\right),
$$

and

$$
C_{7}=C_{4}+C_{2} C_{5} .
$$


Finally, using Eqs. (34) and (42), one gets

$$
\kappa^{2}=C_{8} \exp \left(\frac{C_{7}}{\kappa}\right)
$$

where

$$
C_{8}=\frac{2 C_{6} e^{2} z^{2}}{\varepsilon_{0} \varepsilon_{r} k_{B} T} .
$$

For relatively large-sized ionic impurities (where $\omega_{1} \sim 10^{6}$ $\mathrm{m}^{2} / \mathrm{mol}$ ), EDL thickness of the order of $10 \mathrm{~nm}$ (such that $\kappa \approx$ $10^{8} \mathrm{~m}^{-1}$ ), and low-to-moderate values of fractional coverage of impurities, one will have $C_{7} / \kappa \ll 1$, so that a simplified closed-form expression of $\kappa$ [using Eq. (45)] can be obtained as

$$
\kappa \approx \sqrt{C_{8}} .
$$

However, strictly speaking, one must solve Eq. (45) iteratively to obtain $\kappa$ and from there, for a given value of surface coverage $\beta_{1}$, calculate the surface pressure for the ionic impurities with nonidealities [using Eq. (31)] and the resulting nanobubble contact angle and Laplace pressure.

\section{RESULTS AND DISCUSSIONS}

In this section the significance of the proposed impuritysurface-pressure models [Eqs. (24) and (31)] will be first established by reproducing the experimental results on surfactantinduced variation of the surface tension for simple air-water interfaces of surfactant solutions. The model will be then applied to surface nanobubbles to elucidate the effect of impurities in altering the nanobubble properties. Results will be provided to pinpoint the extent of influence of both the nonionic and ionic nonidealities in the impurity-driven alteration of surface nanobubble properties. The values of the constant parameters used in the present calculation are $\omega_{0}$ (partial molar area of water) $=6.023 \times 10^{4} \mathrm{~m}^{2} / \mathrm{mol}[19,27]$, $R$ (universal gas constant) $=8.314 \mathrm{~J} / \mathrm{mol} \mathrm{K}, T=300 \mathrm{~K}, \varepsilon_{0}=$ $8.8 \times 10^{-12} \mathrm{C} / \mathrm{V}^{2} \mathrm{~m}, \varepsilon=79.8, p_{1} / d_{1}=e$ (where $e$ is the electronic charge).

\section{A. Experimental validation of the present nonideality-based model}

The nonideality-based models connecting the surface pressure to the impurity coverage [Eqs. (24) and (31)] are applicable to any general air-water interface with impurity (surfactant) coverage. In this section the generality of these models are established by demonstrating that these models, with appropriate choices of parameters, can aptly reproduce the experimental results of the surfactant-induced lowering of surface tension. The first step to reproduce the experimental results is to express the surface pressure in terms of the bulk surfactant concentration. This is necessitated by the fact that the experiments invariably report the lowering of surface tension as a function of the concentration in the bulk. As a result one needs to iteratively solve the surface pressure relations [Eqs. (24) and (31)] in conjunction with Eqs. (10) and (33). Similar to our previous study [19], the dimensionless parameter $K_{1}$ [the surface-bulk distribution coefficient of the impurity (surfactant) molecules, representing the relative tendency of the molecules to get adsorbed to a two-dimensional (2D) surface phase from the three-dimensional (3D) bulk phase] connecting the bulk and surface concentration [see Eq. (10)] is used as a fitting parameter during the iterative solution. The idea on the order of magnitude of $K_{1}$ can be made from a measure of the equivalent dimensional parameter $b_{1}$ (having units of liter/mol), typically specified in surfactant literature. As illustrated in Ref. [19], the magnitude of $K_{1}$ is at least one order of magnitude (or even more) higher than $b_{1}$, when $b_{1}$ is expressed in liters/mol. For certain surfactants $b_{1}$ is known directly from the experimental data $[29,39]$, whereas for others it is calculated from the relation $b_{1}=N_{A} v_{m} \exp \left(-\frac{\Delta G_{\text {ads }}}{R T}\right)$ [28], where $v_{m}$ (in liters) is the molecular volume of the surfactants and $\Delta G_{\text {ads }}$ (in $\mathrm{kJ} / \mathrm{mol}$ ) is the molecular free energy of adsorption (well reported in studies of surfactant solutions). For the present case, with a reported (or calculated) value of $b_{1}, K_{1}$ is varied (in a manner such that its order of magnitude is always approximately one order of magnitude higher than $b_{1}$ ) so as to obtain a satisfactory match with the corresponding experimental results (an exactly similar procedure was also adopted in our previous paper [19]).

For nonionic surfactants, we consider two different classes of surfactants. First, we consider $\mathrm{BHBC}_{16}$ (of the general class $\mathrm{BHBC}_{n}$ ) and the results from both the present model as well as our previous model [19] (without nonideality consideration) are compared with the experimental results [see Fig. 2(a)]. In our previous paper [19], we have compared the ideality-based model for nonionic impurities with the same experimental results. Corresponding values of the parameters $K_{1}$ (obtained directly from the corresponding estimate of $b_{1}$ ) and $\omega_{1}$ are obtained from the literature $[40,41]$ (see Table I for details). For the chosen value of $K_{1}$, the results from the present simulation shows a much better fit to the experimental result as compared to our previous model [19]. For the ideality-based model [19] match with the experimental result is obtained for a much higher value of $K_{1}$ [the corresponding plot is not provided here but is provided in Fig. 2(a) of Ref. [19]; rather the corresponding value of $K_{1}$ (called $K_{1 \text {,ideal }}$ ) is mentioned in Table I]. The nonideality-based model for the nonionic impurities is next validated against the experimental results corresponding to octaethylene-glycol- $n$-alkyl ethers $\left(\mathrm{C}_{n} \mathrm{E}_{8}\right)$ for $n=9$ and $n=13$ [see Fig. 2(b)] [29,42]. The corresponding choices of the parameters $K_{1}$ (calculated from the reported values of $\Delta G_{\text {ads }}$ [42]) as well as the values of $\omega_{1}$ (taken from [42]) are mentioned in Table II. Results from both the present model as well as our previous model [19] (without nonideality consideration) are compared with the experimental results. Similar to the previous case, here too, for the chosen value of $K_{1}$ the results from the present simulation shows a much better fit to the experimental result as compared to

TABLE I. Parameters for obtaining the numerical plots of Fig. 2(a). The idea of the partition coefficient $K_{1}$ is obtained from the corresponding $b_{1}$ values provided in $[40,41]$. The partial molar areas $\omega_{1}$ are obtained from $[40,41]$.

\begin{tabular}{ccc}
\hline \hline$K_{1}$ & $K_{1, \text { ideal }}$ & $\omega_{1}\left(\mathrm{~m}^{2} / \mathrm{mol}\right)$ \\
\hline $1.4 \times 10^{7}$ & $1.9 \times 10^{7}$ & $2.5 \times 10^{5}$ \\
\hline \hline
\end{tabular}


TABLE II. Parameters for obtaining the numerical plots of Fig. 2(b). The idea of the partition coefficient $K_{1}$ is obtained from the corresponding $b_{1}$ values calculated from the $\Delta G_{\text {ads }}$ values provide in [40]. The partial molar areas $\omega_{1}$ are obtained from [42].

\begin{tabular}{lccc}
\hline$n$ & $K_{1}$ & $K_{1, \text { ideal }}$ & $\omega_{1}\left(\mathrm{~m}^{2} / \mathrm{mol}\right)$ \\
\hline 9 & $1.7 \times 10^{5}$ & $2.3 \times 10^{5}$ & $4.55 \times 10^{5}$ \\
13 & $2.2 \times 10^{7}$ & $2.7 \times 10^{7}$ & $3.49 \times 10^{5}$ \\
\hline \hline
\end{tabular}

our previous model [19], and the ideality-based model [19] matches the experimental result only for substantially larger $K_{1}$ values (called $K_{1 \text {,ideal }}$; see Table II). Figures 2(a) and 2(b) clearly demonstrate the manner in which the underprediction of the surface pressure, on account of neglect of the nonideal components, necessitates a higher value of $K_{1}$, thereby enforcing a larger value of surface concentration for a given bulk concentration.

For the ionic surfactants, the present model is validated against experimental results corresponding to lithium dodecyl sulphate (LDS) and sodium dodecyl sulphate (SDS) [29,39, 43], [see Fig. 2(c)]. The values of the fitting parameters $K_{1}$ (see Table III) invariably needs to be considerably more than one order of magnitude higher than $b_{1}$ (values of $b_{1}$ for alkali dodecyl sulphate are typically in the range of 40-50 liters/mol [27]), so as to ensure acceptable fit with the experimental results. This is necessitated by the fact that in the present model the contribution of the interaction of hydrophobic heads of the oriented surfactant molecules [35,38] (this effect becomes significantly high for ionic surfactants) are not accounted for. Comparison of the ideality- and nonideality-based models for the case with ionic impurities exhibit that for the said choice of $K_{1}$ the former is largely deviated from the experimental values, for reasons already delineated here [in our previous paper, only when a significantly high value of $K_{1}$ (more than two orders of magnitude higher than $b_{1}$ ) is used, the ideality-based calculations could validate the experimental results corresponding to SDS; these values, $K_{1 \text {,ideal }}$, are shown in Table III]. Thus for both nonionic and ionic impurities, the present nonidealitybased model ensures reproduction of the experimental results with parameter choices that ascertain relatively lesser value of surface impurity coverage, a condition that may be essential for nanobubble systems that are invariably ultraclean containing only trace amounts of impurities. In the following sections this general nonideality-based surface-pressure-surfactant model will be applied to calculate the impurity-induced alterations of the surface nanobubble properties.

TABLE III. Parameters for obtaining the numerical plots of Fig. 2(c). The idea of the partition coefficient $K_{1}$ is obtained from the corresponding $b_{1}$ values obtained directly from [39] or calculated from $\Delta G_{\text {ads }}$ values given in [43]. The partial molar areas $\omega_{1}$ are obtained from [44].

\begin{tabular}{lccc}
\hline \hline Nature of alkali dodecyl sulphate & $K_{1}$ & $K_{1, \text { ideal }}$ & $\omega_{1}\left(\mathrm{~m}^{2} / \mathrm{mol}\right)$ \\
\hline Sodium dodecyl sulphate (SDS) & 2600 & 4000 & $2.85 \times 10^{5}$ \\
Lithium dodecyl sulphate (LDS) & 2400 & 3700 & $2.97 \times 10^{5}$ \\
\hline \hline
\end{tabular}
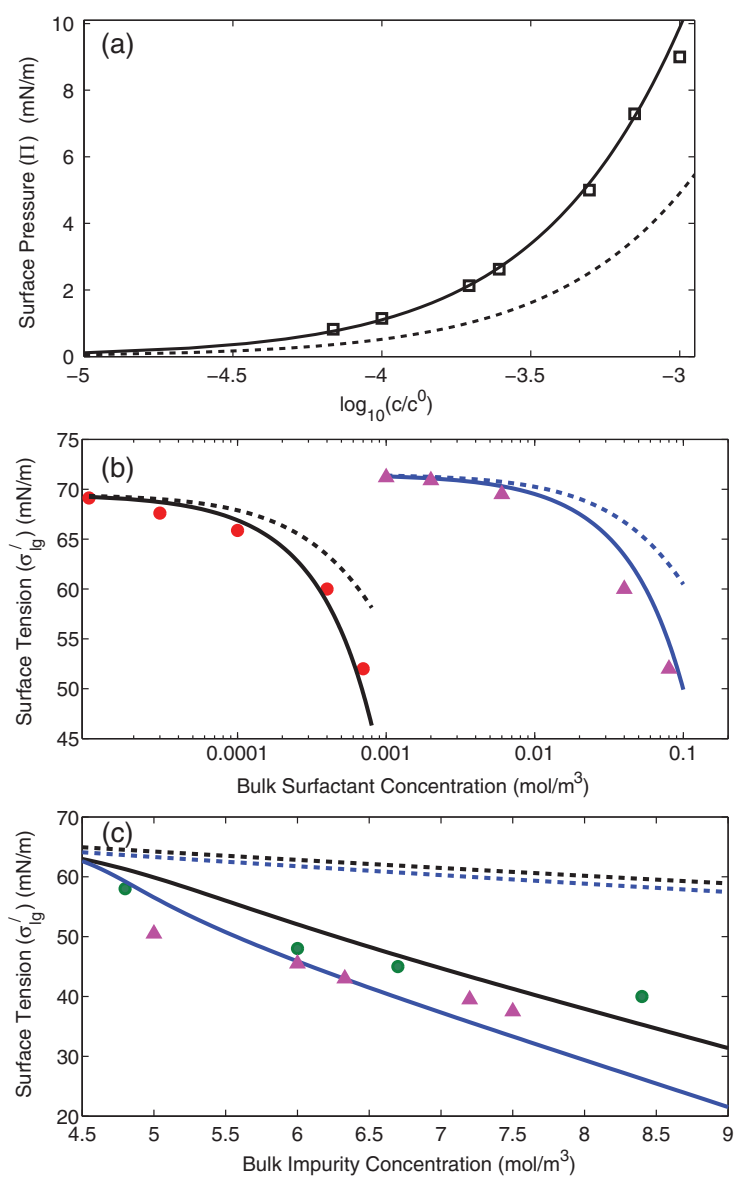

FIG. 2. (Color online) (a) Variation in the surface pressure with bulk ionic concentration ( $c$ is in $\mathrm{mol} / \mathrm{m}^{3}$ and $c_{0}=1 \mathrm{~mol} / \mathrm{m}^{3}$ ) of nonionic surfactants $\mathrm{BHBC}_{16}$. The experimental result (obtained from $[40,41]$ ) is shown by open squares, whereas the simulation result is denoted by continuous lines (the case with nonideality is represented by bold lines whereas that without nonideality is represented by dashed lines). The different parameters used for obtaining the simulation plots are tabulated in Table I. (b) Variation of the surface tension (in $\mathrm{mN} / \mathrm{m}$ ) with bulk concentration of nonionic surfactants octaethylene-glycol- $n$-alkyl ethers $\mathrm{C}_{n} \mathrm{E}_{8}$. Variations corresponding to $n=9$ and $n=13$ are shown. For $n=9$, the experimental results $[29,42]$ are denoted by magenta triangles, whereas the results from the present simulation are shown by the solid blue line. For $n=13$, the experimental results [29,42] are denoted by red circles, whereas the results from the present simulation are shown by the solid black line. For either $n$, the case with nonideality is represented by bold lines whereas that without nonideality is represented by dashed lines. The different parameters used for obtaining the simulation plots are tabulated in Table II. (c) Variation of the surface tension (in $\mathrm{mN} / \mathrm{m}$ ) with bulk concentration of ionic surfactants alkali dodecyl sulphates. For SDS the experimental results $[29,39]$ are denoted by magenta triangles, whereas the results from the present simulation are shown by the solid blue line. For LDS, the experimental results $[29,43]$ are denoted by green circles, whereas the results from the present simulation are shown by the solid black line. For either of the surfactants, the case with nonideality is represented by bold lines whereas that without nonideality is represented by dashed lines. The different parameters used for obtaining the simulation plots are tabulated in Table III. 


\section{B. Effect of nonionic impurities (with nonidealities) on surface nanobubbles}

Figures 3(a) and 3(b) demonstrate the individual and collective contributions of the enthalpic [see Eqs. (17), (20), and (23)] and the entropic nonideality [see Eq. (14)] effects to the surface pressure variation, expressed as functions of partial molar area of the impurities $\left(\omega_{1}\right)$ and the fractional surface coverage of the impurities $\left(\beta_{1}\right)$. Figures 3(a) and 3(b) clearly exhibit that unlike the ideal case [19] (the corresponding value of the ideal surface pressure is provided in the figure caption), for a nonideal air-water interface the surface pressure depends on impurity sizes (characterized by $\omega$ ) even for nonionic impurities. Larger surface coverage $\left(\beta_{1}\right)$, quite intuitively, leads to enhancement of either of the types of nonideality effects [compare Figs. 3 and 3(b)]. However, these effects
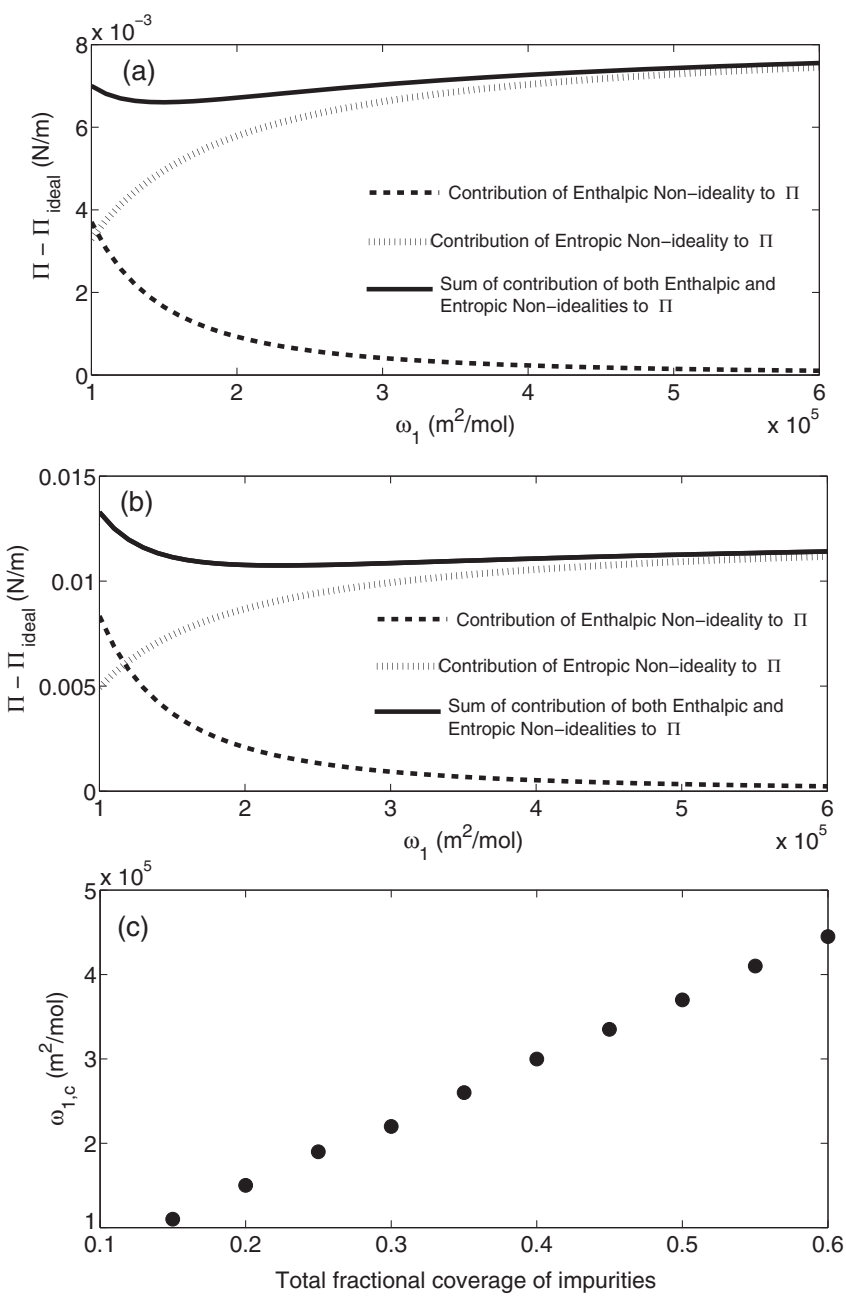

FIG. 3. Variation of the nonideal component of surface pressure with the partial molar area $\left(\omega_{1}\right)$ of the impurity molecules (nonionic) due to individual and combined contributions of the enthalpic and entropic nonidealities for (a) total fractional coverage of impurities $\beta_{1}=0.20$ (for this case $\Pi_{\text {ideal }}=9.2 \times 10^{-3} \mathrm{~N} / \mathrm{m}$ and $\omega_{1, \mathrm{c}}=1.5 \times$ $10^{5} \mathrm{~m}^{2} / \mathrm{mol}$ ) and (b) total fractional coverage of impurities $\beta_{1}=$ 0.3 (for this case $\Pi_{\text {ideal }}=14.8 \times 10^{-3} \mathrm{~N} / \mathrm{m}$ and $\omega_{1, \mathrm{c}}=2.2 \times 10^{5}$ $\mathrm{m}^{2} / \mathrm{mol}$ ). (c) Variation of $\omega_{1, \mathrm{c}}$ with total fractional coverage of impurities $\left(\beta_{1}\right)$. $\omega_{1, \mathrm{c}}$ varies linearly with $\beta_{1}$ (see Appendix for the derivation) with a slope $\sim 6.8 \times 10^{5} \mathrm{~m}^{2} / \mathrm{mol}$. demonstrate opposite dependencies on the partial molar area of the impurities $\omega_{1}$. For a given value of $\beta_{1}$, increase in $\omega_{1}$ (or the impurity size) reduces the enthalpic effects, but augments the entropic nonidealities. Smaller $\omega_{1}$, for a given value of impurity coverage, implies a larger number of impurity molecules at the air-water interface, triggering a larger number of possible dipole-dipole interactions, thereby enhancing the enthalpic effects. On the contrary, larger-sized impurity molecules lead to a more effective expelling of the smaller water molecules from their vicinity at the air-water interface causing an enhanced effective intermixing of the impurity molecules and hence augmented entropic effects [Eq. (14) clearly quantifies such a size dependence of the entropic nonidealities]. The opposite dependencies of the enthalpic and entropic effects on impurity size ensure that the surface pressure first decreases (implicating a dominant influence of the enthalpic effects), attains a minimum (the corresponding value of $\omega_{1}$ is $\left.\omega_{1, \mathrm{c}}\right)$, and then increases (implicating a dominant influence of the entropic effects) with impurity sizes. Enthalpic effects increase nonlinearly with the surface coverage, whereas the entropic effects exhibit a linear dependence on the same [see Eq. (24)]. Consequently for larger $\beta_{1}$ the dominance of enthalpic effects persists over larger ranges of impurity sizes and hence [see Figs. 3(a) and $3(\mathrm{c})]$ the value of $\omega_{1, \mathrm{c}}$ is found to increase linearly with $\beta_{1}$ (see Appendix for the mathematical demonstration).

Figures 4(a)-4(c) demonstrate the individual and collective impact of the enthalpic and the entropic nonidealities, in conjunction with the ideal effects, in varying the nanobubble (gas-side) contact angle $\theta$ as functions of $\omega_{1}$ and $\beta_{1}$. For substantially small $\omega_{1}$, enthalpic effects are comparable to the entropic effects and their results lead to a significant decrease of the contact angle. For larger $\omega_{1}$, however, the nonidealities are governed entirely by the entropic effects and the overall contribution of the nonideality effects (in lowering the contact angle) is weakened. Thus it can be inferred that at the $\omega_{1}$ value, where the entropic and enthalpic effects are relatively comparable, one gets the maximum impact of nonidealities. The most important statement of Figs. 4(a)-4(c) is that for suitable values of the impurity sizes, the nonideality effects can lead to a substantial lowering of the contact angle (making it match with the experimental results $[2,7,20]$ ) at an impurity surface coverage which is substantially lower than that for the case without the nonideality effects (e.g., with nonideality effects with $\omega_{1}=1.2 \mathrm{~mol} / \mathrm{m}^{2}, \theta \sim 20^{\circ}$ for $\beta_{1} \sim 0.4$, whereas without nonideality effects, $\theta \sim 20^{\circ}$ for $\beta_{1} \sim 0.8$ [19]). Such a result acts as a very useful starting point emphasizing the fact that consideration of nonideality effects ensures that the effect of impurities (in altering the surface nanobubble parameters) are witnessed at a much lesser concentration as compared to the ideal case. As most of the experiments on detection of surface nanobubbles are performed in an ultraclean environment, one expects a substantially small concentration of surface impurities and in this light the modifications to the ideal scenario, introduced by the appropriate representation of the nonideality effects, become extremely essential in correct representation of the role of the impurities in altering the surface nanobubble properties. However, it must be stated here that the extent of magnification of the impurity effect as evidenced by Figs. 4(a)-4(c), may 

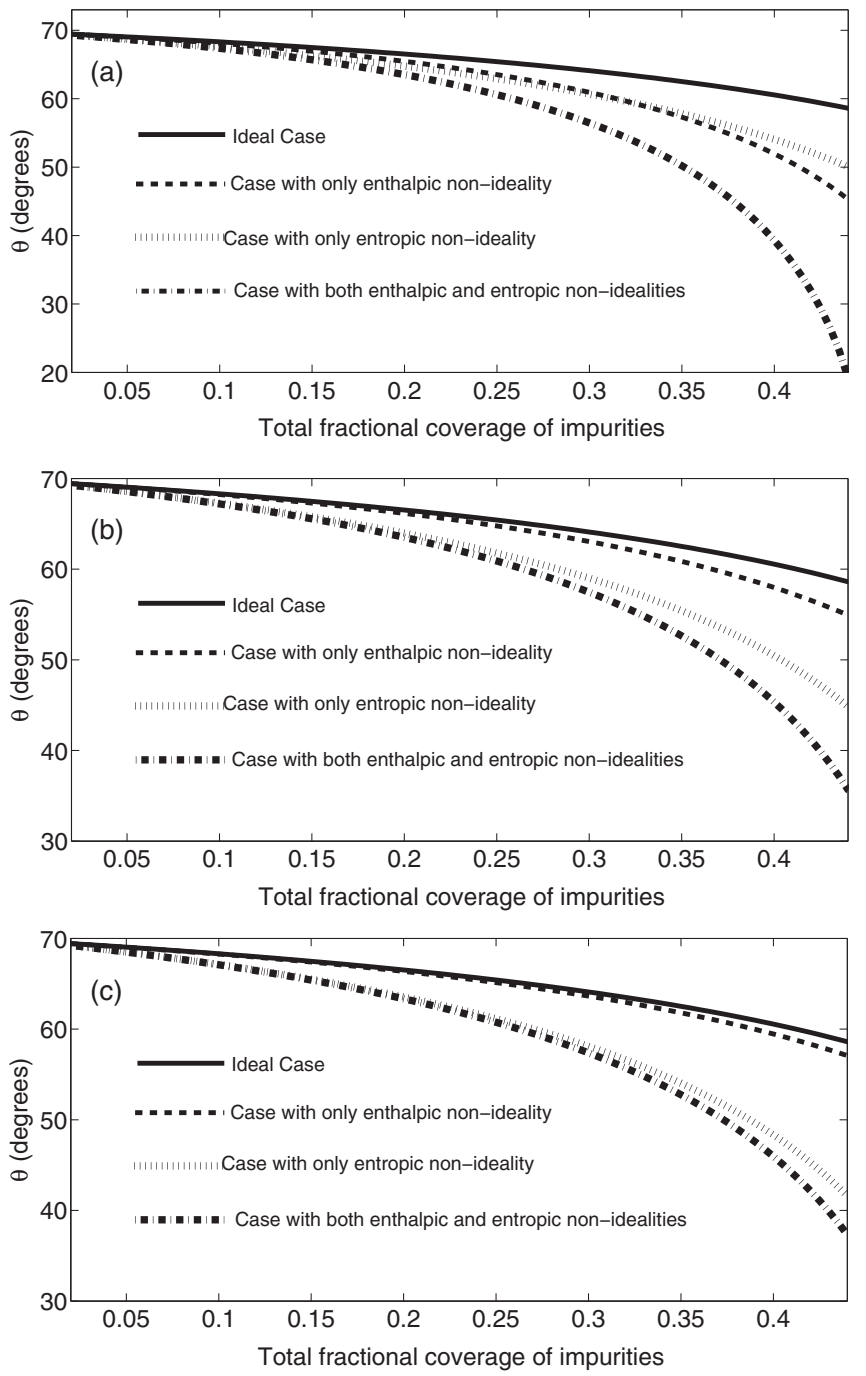

FIG. 4. Variation of gas-side contact angle $\theta$ (obtained from the relation $\cos \theta=\left(\sigma_{s l}-\sigma_{s g}\right) / \sigma_{\mathrm{lg}}^{\prime}$, with $\sigma_{s l}-\sigma_{s g}=\Delta \sigma=0.025 \mathrm{~N} / \mathrm{m}$ and $\left.\sigma_{\mathrm{lg}}^{\prime}=\sigma_{\mathrm{lg}}-\Pi\right)$ with the total fractional coverage of impurities for nonionic impurities (for the situation where there is only one kind of impurity, which is nonionic, at the air-water interface) for cases with and without different types of nonidealities (entropic and enthalpic) for (a) $\omega_{1}=1.2 \times 10^{5} \mathrm{~mol} / \mathrm{m}^{2}$, (b) $\omega_{1}=2 \times 10^{5} \mathrm{~mol} / \mathrm{m}^{2}$, and (c) $\omega_{1}=3 \times 10^{5} \mathrm{~mol} / \mathrm{m}^{2}$.

not be sufficient to completely explain the impurity-induced changes of nanobubble contact angles even at trace amounts of bulk concentration of impurities.

In Figs. 5(a)-5(c), the variation of $\Delta p$ is considered and a similar qualitative effect, as the case with contact angle (the effect of nonideality is maximum for small $\omega_{1}$ ), is observed. It can be seen that $\Delta p$, for small enough $\omega_{1}$, can be as small as $0.5 \mathrm{MPa}$. This is close to one order decrease of the Laplace pressure, though the value is still substantially large to forbid satisfactory explanation of the nanobubble superstability.

\section{Effect of ionic impurities (with nonidealities) on surface nanobubbles}

Section II B illustrates that the effect of ionic nonideality depends on the inverse Debye length $(\kappa)$. Consequently, in
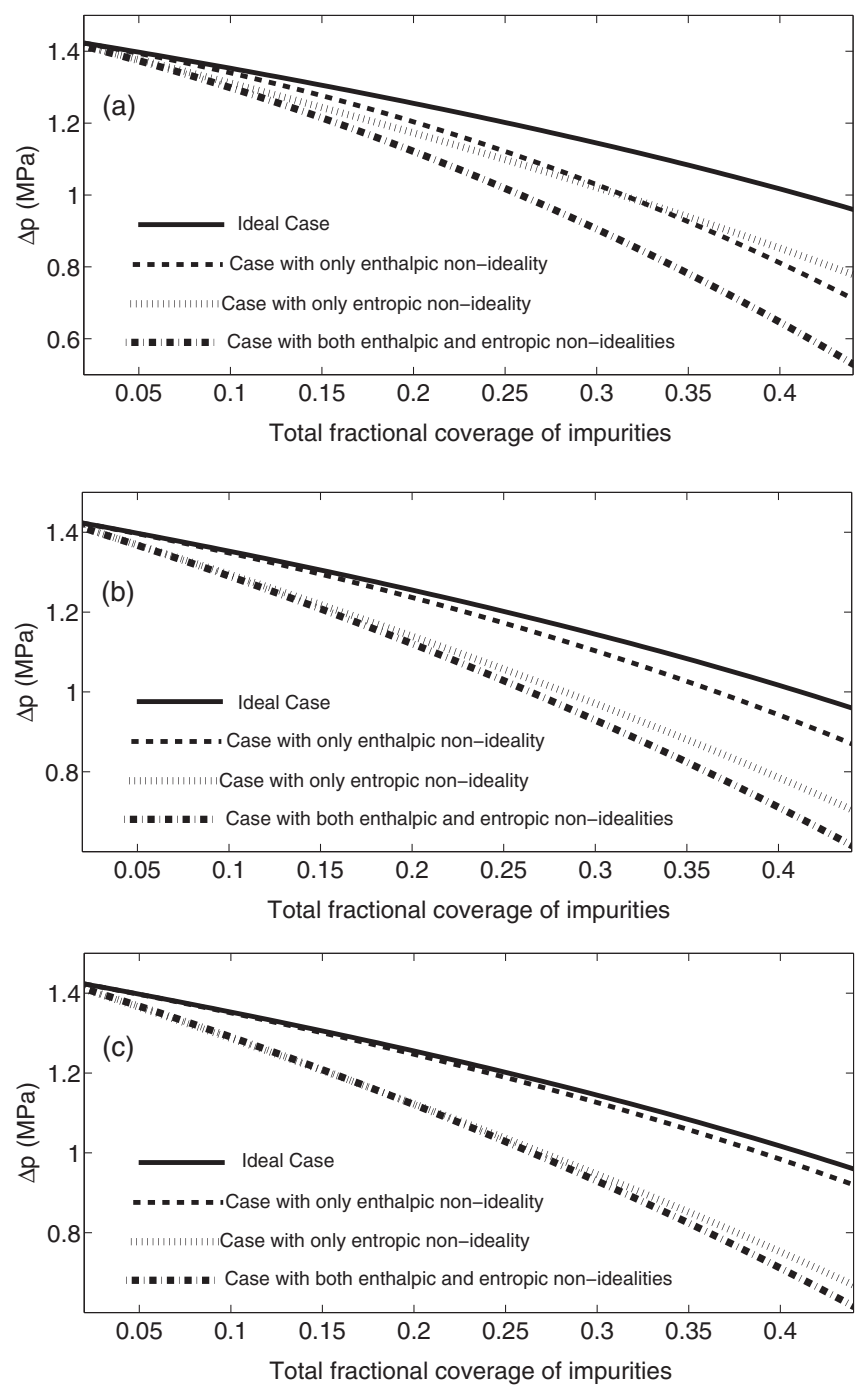

FIG. 5. Variation of Laplace pressure $\Delta p$ (calculated as $\Delta p=$ $2 \sigma_{\mathrm{lg}}^{\prime} / R_{b}$, where $\sigma_{\mathrm{lg}}^{\prime}=\sigma_{\mathrm{lg}}-\Pi$ and the radius of the spherical cap $R_{b}=100 \mathrm{~nm}$ ) with the total fractional coverage of impurities for nonionic impurities (for the situation where there is only one kind of impurity, which is nonionic, at the air-water interface) for cases with and without different types of nonidealities (entropic and enthalpic) for (a) $\omega_{1}=1.2 \times 10^{5} \mathrm{~mol} / \mathrm{m}^{2}$, (b) $\omega_{1}=2 \times 10^{5} \mathrm{~mol} / \mathrm{m}^{2}$, and (c) $\omega_{1}=3 \times 10^{5} \mathrm{~mol} / \mathrm{m}^{2}$.

addition to the parameters such as $\beta_{1}$ and $\omega_{1}$, the effect of bulk concentration of the impurities (or the dimensionless surface-bulk distribution or partition coefficient $K_{1}$ relating the bulk concentration to the surface coverage) becomes central to estimating the impurity-induced changes in surface pressure. In Figs. 6(a) and 6(b), we attempt to bring out such important parametric dependences of $\kappa$. As pointed our earlier, for the present case it is equivalent to either solve for the bulk concentration or the inverse Debye length $\kappa$ (and from there derive the required variables) with a given value of surface coverage $\beta_{1}$, or solve for $\beta_{1}$ with a given value of the bulk concentration. Thus, unlike the cases of ideal air-water interface [19] or nonideal air-water interface with no ionic effects (see Secs. II A and III B), for the present case additional simplification is not possible by expressing 

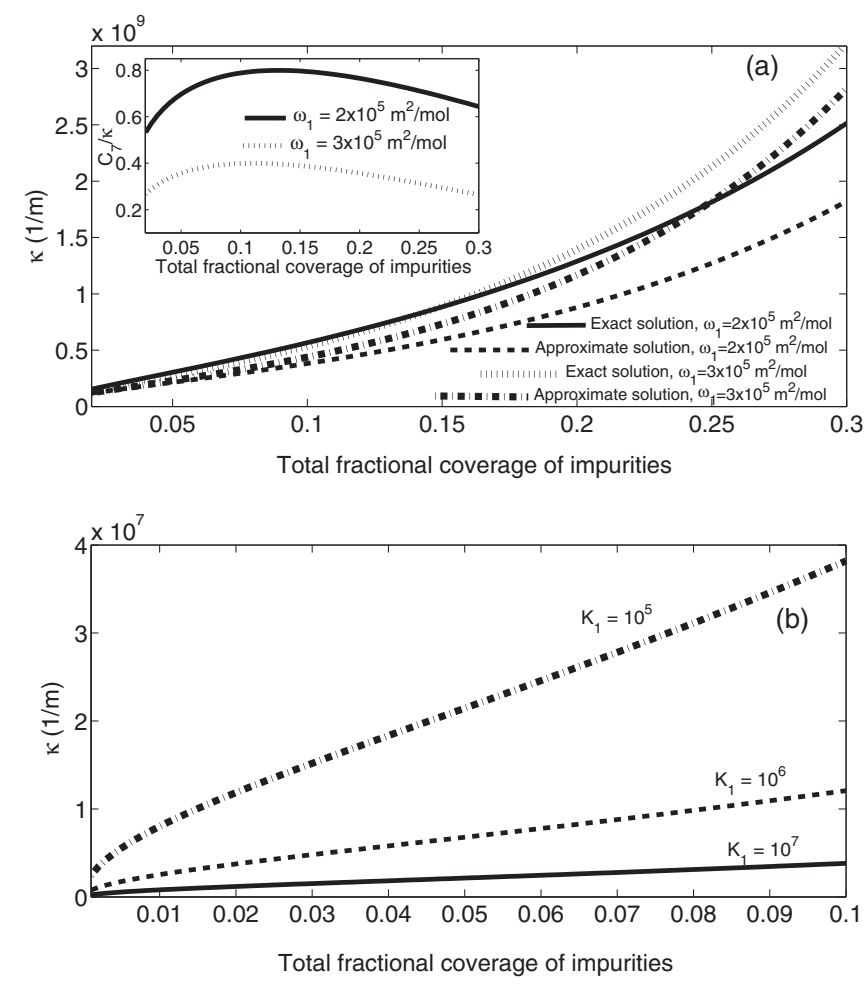

FIG. 6. (a) Exact and approximate solutions for the variation of the inverse Debye length $(\kappa)$ [exact solution is obtained by iteratively solving Eq. (45), whereas the approximate solution is obtained from Eq. (47)] with the total fractional coverage of impurities for ionic impurities for different values of $\omega_{1}$ for partition coefficient $K_{1}=$ $10^{3}$. Inset: The corresponding variation of the ratio $C_{7} / \kappa$ [with $\kappa$ obtained from the exact solution of Eq. (45)] with the total fractional coverage of impurities. (b) Variation of inverse Debye length $(\kappa)$ [we consider the exact solution of $\kappa$ obtained by iteratively solving Eq. (45)] with the total fractional coverage of impurities for ionic impurities for higher ranges of values of partition coefficient $K_{1}$. Solutions are provided for $\omega_{1}=2 \times 10^{5} \mathrm{~mol} / \mathrm{m}^{2}$.

the surface pressure solely in terms of the surface coverage of impurities. In fact most of the studies on effects of ionic nonidealities on surface pressure $[29,38]$ calculate the surface coverage and the surface pressure from a known value of bulk concentration. However, in the present calculation we adhere to the approach where the value of surface coverage $\left(\beta_{1}\right)$ is specified and other parameters are accordingly calculated. This ensures efficient representation of the comparative influence of the ionic nonidealities, with respect to the other nonideality effects, in affecting the surface pressure. Figure 6(a) describes the exact [see Eq. (45)] and the approximate [see Eq. (47)] solutions of $\kappa$, expressed as functions of $\beta_{1}$ and $\omega_{1}$ for a given $K_{1}$. At the inset of Fig. 6(a), the corresponding variation of the parameter $C_{7} / \kappa$ is provided, and it acts as a measure to quantify the extent of difference between the exact and the approximate solutions. With $C_{7} / \kappa \ll 1$, these two solutions show nice agreement. However they differ significantly for larger $C_{7} / \kappa . \kappa$ is found to increase with the fractional coverage of impurities $\left(\beta_{1}\right)$ at the air-water interface. Larger $\beta_{1}$, for a given value of partition coefficient $K_{1}$, implies a larger bulk concentration of the ionic impurities, thereby justifying a smaller screening length (or larger $\kappa$ ). Again for a given $\beta_{1}$, larger sizes of ionic impurities (i.e., larger $\omega_{1}$ ) at the air-water interface imply a lesser number of impurity molecules causing a lesser number of ion-ion interactions. The difference between the exact and approximate solutions of $\kappa$ primarily stems from the accurate representation of the ion-ion interactions, and accordingly larger $\omega_{1}$ leads to a lesser difference between these two solutions [see Fig. 6(a)]. The consequence of partition coefficient $K_{1}$ on $\kappa$ is manifested in Fig. 6(b). A larger partition coefficient implies larger surface coverage for a given bulk concentration, or weaker bulk concentration (and hence larger screening length or smaller $\kappa)$ for a given surface coverage $\left(\beta_{1}\right)$. The partition coefficient $K_{1}$ signifies the relative tendency of the impurity molecules to occupy the two-dimensional surface state from the threedimensional liquid bulk state and there are several studies [44-46], which confirm that the partition coefficient values chosen in Fig. 6(b) can be expected for various surfactant molecules.

Figures 7(a)-7(c) detail the contribution of each type of nonideality effects [entropic, enthalpic (nonionic), and ionic] in the variation of the surface pressure for different combinations of the parameter values. Figures 7(a) and 7(b) demonstrate these individual contributions for a relatively small value of $K_{1}$. It is witnessed that in sharp contrast to the enthalpic and entropic effects, the surface pressure due to ionic effects does not increase with $\beta_{1}$ monotonically. Rather, it first increases, attains a maximum and then decreases with $\beta_{1}$. Larger $\kappa$ or smaller screening length (at larger $\beta_{1}$ ) causes the ionic interactions (cause of the nonideal ionic surface pressure) to be present only over reduced length scales, which decreases its net effect [as quantified through $1 / \kappa$ dependence of surface pressure; see Eq. (31)]. At the same time, however, an increase in $\beta_{1}$ will lead to a higher number of ion-ion interactions, thereby enhancing the surface pressure. For smaller ranges of $\beta_{1}$, such an increase in the number of ion-ion interactions overwhelms the effect of a corresponding increase in $\kappa$ (with $\beta_{1}$ ), thereby making the surface pressure increase with $\beta_{1}$. However, nonlinear dependence of $\kappa$ on $\beta_{1}$ ensures that for higher $\beta_{1}$, the effect of increase in $\kappa$ (with $\beta_{1}$ ) is more dominant causing a net lowering of ionic nonideal surface pressure with $\beta_{1}$. Increase in impurity size will lead to a lesser number of ion-ion interactions (as already discussed above), causing a progressive lowering of the ionic nonideality contribution to surface pressure [see Figs. 7(a) and 7(b)]. Figure 7(c) demonstrates the individual contribution of the different nonideality effects for larger ranges of $K_{1}$. It is clearly revealed that at such $K_{1}$ values the lowering of the inverse EDL thickness $\kappa$ (attributable to the fact that at such a high $K_{1}$ value, most of the impurity ions prefer to be localized at the air-water interface, causing a large lowering of the bulk concentration leading to substantially weak EDL screening with larger screening length or smaller $\kappa$ ) is so significant that the ionic nonideality effect completely dominates the nonionic nonideality effects and leads to substantial lowering of the surface tension for extremely small values of surface coverage of impurities. In fact the surface coverage (for relevant increases of surface pressure $\sim 0.05 \mathrm{~N} / \mathrm{m}$ with ionic nonidealities) remains so small that one witnesses only the monotonic increase of the surface pressure with the surface 

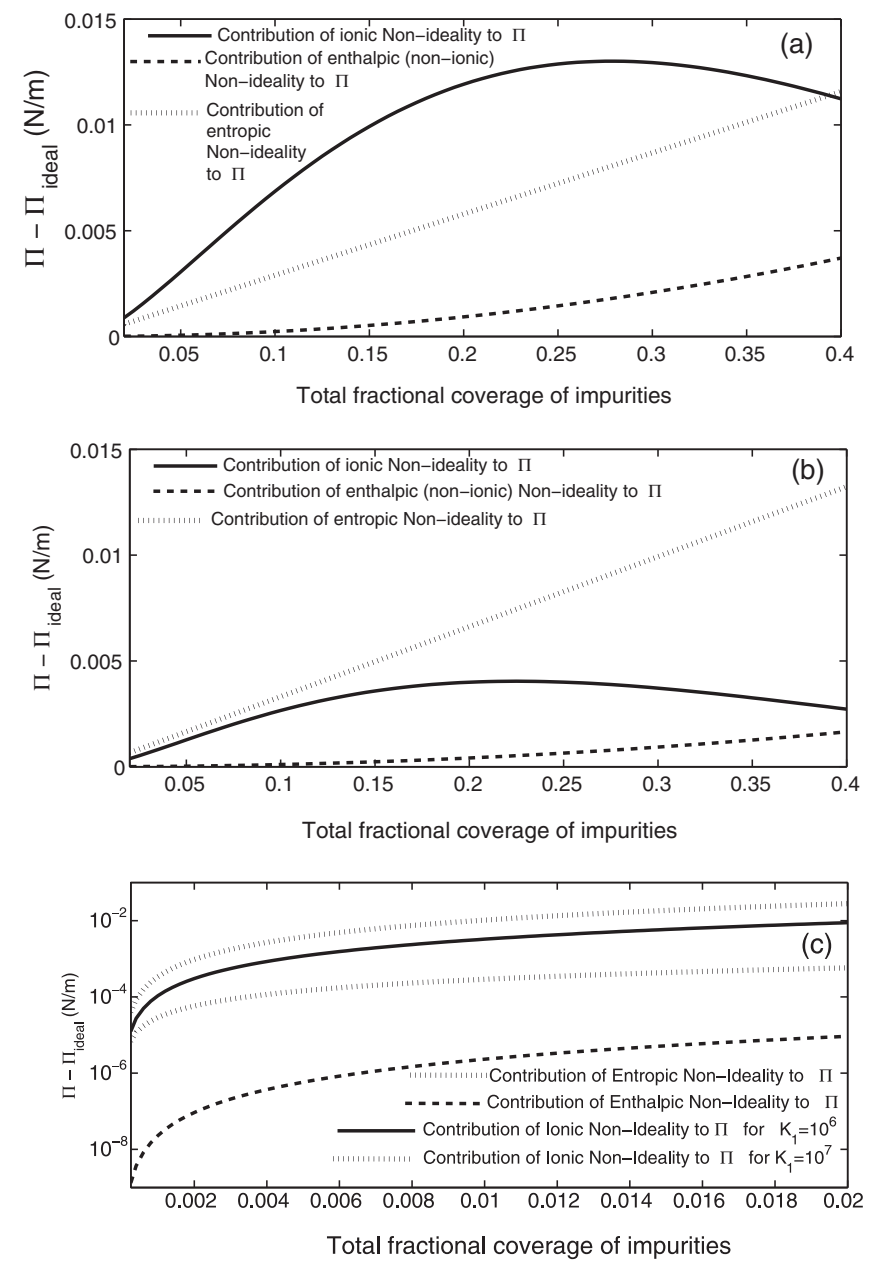

FIG. 7. Variation of the nonideal components of surface pressure [namely ionic, enthalpic (nonionic), and entropic components] with total fractional coverage of impurities (for the situation where there is only one kind of impurity at the air-water interface and that impurity is ionic in nature, with all the impurity ions acting as potential determining ions) for (a) $\omega_{1}=2 \times 10^{5} \mathrm{~mol} / \mathrm{m}^{2}$ and (b) $\omega_{1}=3 \times 10^{5} \mathrm{~mol} / \mathrm{m}^{2}$ (for the case with ionic nonideality effects, we consider an exact solution of $\kappa$ with $K_{1}=10000$ ). (c) Variation of these nonideal components shown for larger $K_{1}$ values at $\omega_{1}=$ $2 \times 10^{5} \mathrm{~mol} / \mathrm{m}^{2}$. At such large values of $K_{1}$ the ionic nonideality contribution is significantly higher than the nonionic nonideality contributions (which are independent of $K_{1}$ ), leading to substantial increase in the surface pressure even for very weak surface coverage. Consequently the plots are shown only over much smaller ranges of surface coverage.

coverage [similar to those seen in Figs. 7(a) and 7(b) for ionic nonideality for smaller surface coverage].

Figures 8(a)-8(c) depict the variations in nanobubble contact angle $\theta$ with fractional coverage of impurities, portraying the individual and collective consequences of the ionic and nonionic [nonionic effects consist of entropic and enthalpic (nonionic) components] nonideality effects for different ranges of parameter values. Figures 8(a) and 8(b) depict the contact angle variation for relatively small values of $K_{1}$. Smaller $\omega_{1}$ leads to a large number of identically charged ions at the air-water interface enforcing a large expenditure of surface energy so as to allow the similarly charged ions to remain in
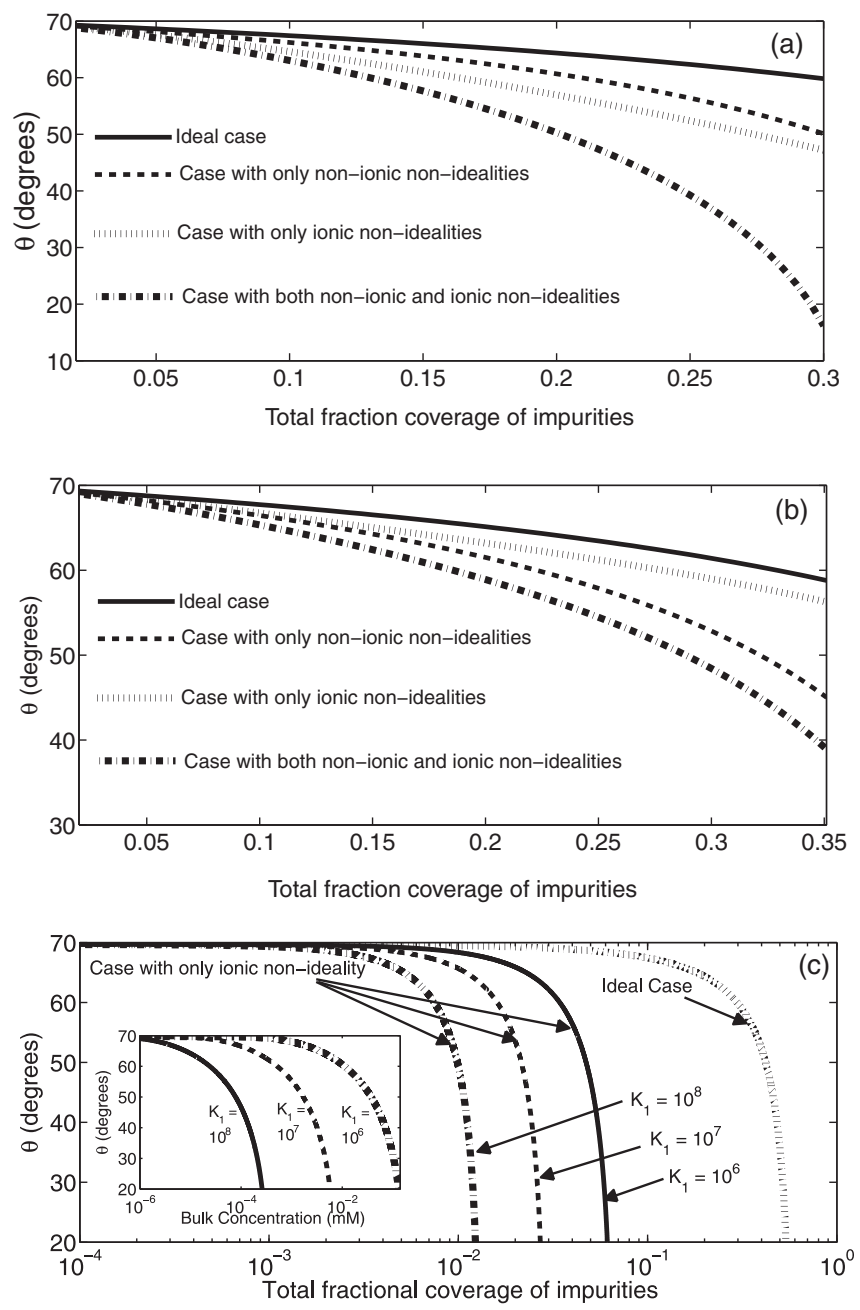

FIG. 8. Variation of gas-side contact angle $\theta$ (obtained from the relation $\cos \theta=\left(\sigma_{s l}-\sigma_{s g}\right) / \sigma_{\mathrm{lg}}^{\prime}$, with $\sigma_{s l}-\sigma_{s g}=\Delta \sigma=0.025 \mathrm{~N} / \mathrm{m}$ and $\sigma_{\mathrm{lg}}^{\prime}=\sigma_{\mathrm{lg}}-\Pi$ ) with the total fractional coverage of impurities for ionic impurities for ideal and nonideal (different types of nonideality effects are considered separately as well as simultaneously) air-water interface (for the situation where there is only one kind of impurity at the air-water interface and that impurity is ionic in nature, with all the impurity ions acting as potential determining ions) for (a) $\omega_{1}=2 \times 10^{5} \mathrm{~mol} / \mathrm{m}^{2}$ and (b) $\omega_{1}=3 \times 10^{5} \mathrm{~mol} / \mathrm{m}^{2}$ (for the case with ionic nonideality effects, we consider an exact solution of $\kappa$ with $K_{1}=10000$ ). (c) Variation of the gas-side contact angle $\theta$ for larger $K_{1}$ values at $\omega_{1}=2 \times 10^{5} \mathrm{~mol} / \mathrm{m}^{2}$. At such large values of $K_{1}$ the ionic nonideality contribution is so substantial that the case with only ionic nonideality is identical to the case with both ionic and nonionic nonidealities. The corresponding variations of the nonionic nonidealities are already shown in Fig. 7(a). We also provide the contact angle variation for the ideal case. In the inset of the figure we provide the variation of the contact angle as a function of the corresponding bulk concentration of the ionic impurities for different $K_{1}$ values.

the air-water interface despite their strong mutual repulsion. This ensures significant contribution of the ionic nonideality effects to the surface pressure. Hence for smaller $\omega_{1}$, the ionic nonideality contribution (in lowering $\theta$ ) dominates, whereas for larger $\omega_{1}$ an augmented contribution of the entropic nonideality effects [see Figs. 3(a) and 3(b)] makes 

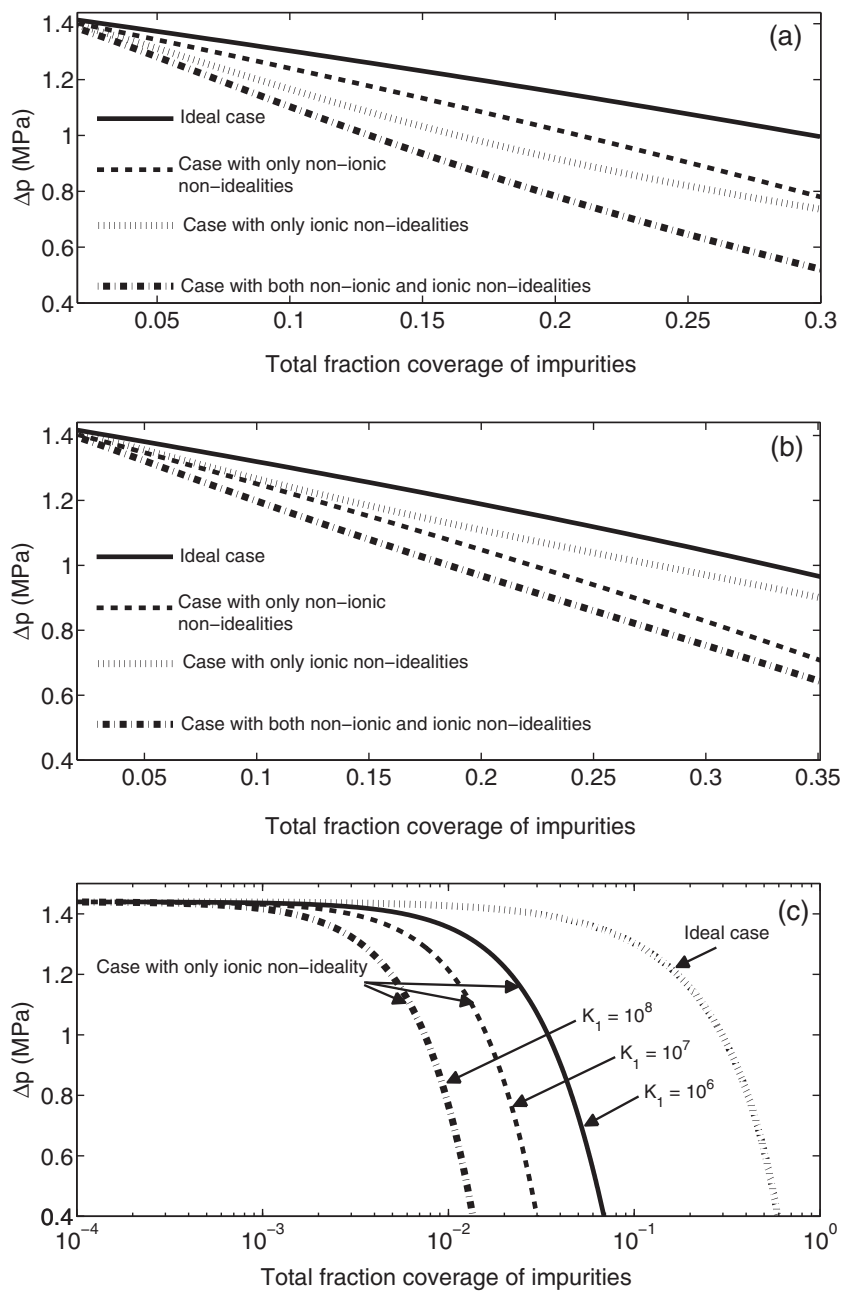

FIG. 9. Variation of Laplace pressure $\Delta p$ (calculated as $\Delta p=$ $2 \sigma_{\mathrm{lg}}^{\prime} / R_{b}$, where $\sigma_{\mathrm{lg}}^{\prime}=\sigma_{\mathrm{lg}}-\Pi$ and the radius of the spherical cap $R_{b}=100 \mathrm{~nm}$ ) with the total fractional coverage of impurities for ionic impurities for ideal and nonideal (different types of nonideality effects are considered separately as well as simultaneously) air-water interface (for the situation where there is only one kind of impurity at the air-water interface and that impurity is ionic in nature, with all the impurity ions acting as potential determining ions) for (a) $\omega_{1}=2 \times 10^{5} \mathrm{~mol} / \mathrm{m}^{2}$ and (b) $\omega_{1}=3 \times 10^{5} \mathrm{~mol} / \mathrm{m}^{2}$ (for the case with ionic nonideality effects, we consider an exact solution of $\kappa$ with $K_{1}=$ $10000)$. (c) Variation of Laplace pressure $\Delta p$ for larger $K_{1}$ values at $\omega_{1}=2 \times 10^{5} \mathrm{~mol} / \mathrm{m}^{2}$. At such large values of $K_{1}$ the ionic nonideality contribution is so substantial that the case with only ionic nonideality is identical to the case with both ionic and nonionic nonidealities. The corresponding variations of the nonionic nonidealities are already shown in Fig. 8(a). We also provide the Laplace pressure $\Delta p$ variation for the ideal case.

the contribution due to the nonionic effects (in lowering $\theta$ ) larger. When these effects are simultaneously considered, the nanobubble contact angles are found to attain values that match well with the experimental results $[2,7,20]$ for as low a fraction of surface coverage as $0.25-0.3$. Figure $8(\mathrm{c})$ portrays the variation of the contact angle for larger ranges of $K_{1}$ values. The remarkably large lowering of $\kappa$ at such $K_{1}$ values ensure that the nanobubble contact angle attains the experimentally observed values at a surface coverage which is one to two orders smaller than that predicted for the ideal case. Such a remarkable lowering of surface coverage has not been witnessed for cases with other types of nonideality effects or at smaller $\mathrm{K}_{1}$ [see Figs. 4(a)-4(c) and 8(a) and 8(b)]. An even more stunning effect is witnessed if one plots [see inset of Fig. 8(c)] the contact angle as a function of the corresponding bulk ionic concentration [we use Eqs. (10) and (32) to obtain the corresponding bulk concentration]. This figure shows that with suitable choice of $K_{1}$ (note such augmented partition coefficient $K_{1}$ are very commonplace; see refs. [44-46]), one can ensure that nanobubble contact angles can match the experimental values for as low a bulk concentration as just few micromolars or even less. Such trace amounts of impurities in the bulk are common even in ultraclean experimental setups studying surface nanobubbles [8], and in this light our present theory indeed satisfactorily explains how the impurities, even in very minute bulk concentration, may substantially affect the surface nanobubble contact angles. Also note that Fig. 8(c) provides a direct idea on the nature of the impurities that should be present in nanobubble experimental setup. Such impurities must have a partition coefficient substantially large so that they will exhibit a great propensity to be adsorbed at the air-water interface of the surface nanobubble, allowing them to impart a large influence in lowering the nanobubble contact angle even when their bulk concentration is substantially small.

In Figs. 9(a)-9(c) the corresponding variations in $\Delta p$ are depicted for different ranges of parameter values as well the combination of ionic and nonionic nonideality effects. Under optimized combination of the parameters one can kind find substantially small Laplace pressure. However, the Laplace pressure still remains significantly high to forbid stability. Thus the present paper can mainly be viewed as the one that sheds light on the possible role of impurities in affecting the contact angle of surface nanobubbles, but not the nanobubble superstability.

\section{CONCLUSIONS}

In this paper our previous ideality-based model [19] on the effect of impurities on surface nanobubbles is extensively elaborated by incorporating the consequences of ionic and nonionic nonidealities stemming from finite interactions between impurity molecules in a nonideal air-water interface. It is demonstrated that with the appropriate accounting of the nonideality effects the impurity-induced altered values of the nanobubble contact angle match well with the experimental results $[2,7,20]$ at a surface coverage which is significantly smaller than that predicted by the ideality-based model [19]. In fact, our theory demonstrates that for impurities with a substantially large partition coefficient the lowering of the nanobubble contact angle to experimentally observed values can be achieved with bulk impurity concentration in the order of few micromolars or even less. The above findings, in light of the fact that the ultraclean experimental conditions for detecting surface nanobubbles will only lead to trace amounts of impurities, makes this newly proposed nonideality-based model extremely important in the context of surface nanobubble studies. This model provides an important theoretical clue (something which was impossible with our previous simplified model [19]) on how even a very small bulk 
concentration of impurities leads to substantial lowering of nanobubble contact angle. The impurity effects are also found to reduce the Laplace pressure, but the latter still remains substantially high to forbid stability. Thus we do not claim to have solved the surface nanobubble superstability mystery. Rather through the present and the previous [19] models, we have provided conclusive quantitative evidence on the prominent role of the impurities in ensuring the anomalously small contact angles of surface nanobubbles.

\section{ACKNOWLEDGMENTS}

The author sincerely acknowledges Professor Detlef Lohse and Dr. Jacco H. Snoeijer of Physics of Fluids, University of Twente, for carefully reading the manuscript and providing invaluable suggestions that have been extremely useful in improving the manuscript.

\section{APPENDIX: DERIVATION OF THE RELATION BETWEEN $\omega_{1, \mathrm{c}}$ AND $\beta_{1, \mathrm{c}}$}

The surface pressure $\Pi$ for nonionic impurities (with nonideal effects) is a joint function of the variables $\omega_{1}$ and $\beta_{1}$ (for the case with one impurity). As a result the signs of the quantities $\frac{\partial^{2} \Pi}{\partial \beta_{1}^{2}}, \frac{\partial^{2} \Pi}{\partial \omega_{1}^{2}}$ and $D=\frac{\partial^{2} \Pi}{\partial \beta_{1}^{2}} \frac{\partial^{2} \Pi}{\partial \omega_{1}^{2}}-\left(\frac{\partial^{2} \Pi}{\partial \omega_{1} \partial \beta_{1}}\right)^{2}$, evaluated at the critical points (obtained by setting $\frac{\partial \Pi}{\partial \beta_{1}}=0$ or $\frac{\partial \Pi}{\partial \omega_{1}}=0$ ) need to be checked to decide the nature of the extremum.

With $\frac{\partial \Pi}{\partial \omega_{1}}=0$, one gets [from Eq. (24)] the condition at the critical point as

$$
\omega_{1, c}=\frac{2 S_{1}}{R T} \beta_{1, c}
$$

where

$$
S_{1}=\frac{1}{\left(4 \pi \varepsilon_{0} \varepsilon_{r}\right)^{2}} \frac{2 \pi N_{A}^{2}}{5 k_{B} T} \frac{p_{1}^{4}}{d_{11}^{4}} .
$$

Also from Eq. (24), one can obtain

$$
\frac{\partial^{2} \Pi}{\partial \beta_{1}^{2}}=\frac{R T}{\omega_{0}} \frac{1}{\left(1-\beta_{1}\right)^{2}}+2 S_{1} \omega_{1}^{2}>0,
$$

(as $S_{1}$ is always positive).

Similarly from Eq. (24),

$$
\frac{\partial^{2} \Pi}{\partial \omega_{1}^{2}}=\frac{\beta_{1}}{\omega_{1}^{3}}\left[\frac{6 S_{1} \beta_{1}}{\omega_{1}}-2 R T\right] .
$$

Using Eq. (A1) in Eq. (A4), one gets

$$
\left(\frac{\partial^{2} \Pi}{\partial \omega_{1}^{2}}\right)_{\beta_{1, c}, \omega_{1, c}}=\frac{R^{2} T^{2}}{S_{1} \omega_{1, c}^{2}}>0 .
$$

The mixed derivative can be calculated as [from Eq. (24)]:

$$
\frac{\partial^{2} \Pi}{\partial \omega_{1} \partial \beta_{1}}=\frac{R T}{\omega_{1}^{2}}-\frac{4 S_{1} \beta_{1}}{\omega_{1}^{3}} .
$$

Using Eq. (A1) in Eq. (A6), one gets

$$
\left(\frac{\partial^{2} \Pi}{\partial \omega_{1} \partial \beta_{1}}\right)_{\beta_{1, c}, \omega_{1, c}}=-\frac{R T}{\omega_{1, c}^{2}} .
$$

Hence one finally ends up with

$$
\begin{aligned}
D & =\left[\frac{\partial^{2} \Pi}{\partial \beta_{1}^{2}} \frac{\partial^{2} \Pi}{\partial \omega_{1}^{2}}-\left(\frac{\partial^{2} \Pi}{\partial \omega_{1} \partial \beta_{1}}\right)^{2}\right]_{\beta_{1, c}, \omega_{1, c}} \\
& =\frac{R T}{\omega_{0}} \frac{1}{\left(1-\beta_{1, c}\right)^{2}}>0 .
\end{aligned}
$$

As at $\beta_{1, \mathrm{c}}$ and $\omega_{1, \mathrm{c}}$, one has $D 0, \frac{\partial^{2} \Pi}{\partial \beta_{1}^{2}}>0$, and $\frac{\partial^{2} \Pi}{\partial \omega_{1}^{2}}>0$, it can be inferred that the extremum is a minimum. Also Eq. (A2) establishes that the critical points (or the values of $\beta_{1}$ and $\omega_{1}$ where the function attains minimum) are linearly related, with the slope of this linear variation (with the chosen values of the parameter) being calculated as $6.8 \times 10^{5} \mathrm{~m}^{2} / \mathrm{mol}$.
[1] S. Lou, Z. Ouyang, Y. Zhang, X. Li, J. Hu, M. Li, and F. Yang, J. Vac. Sci. Technol. B 18, 2573 (2000).

[2] N. Ishida, T. Inoue, M. Miyahara, and K. Higashitani, Langmuir 16, 6377 (2000).

[3] G. E. Yakubov, H.-J. Butt, and O. I. Vinogradova, J. Phys. Chem. B 104, 3407 (2000).

[4] A. Carambassis, L. C. Jonker, P. Attard, and M. W. Rutland, Phys. Rev. Lett. 80, 5357 (1998).

[5] J. W. G. Tyrrell and P. Attard, Phys. Rev. Lett. 87, 176104 (2001).

[6] A. C. Simonsen, P. L. Hansen, and B. Klosgen, J. Colloid Interface Sci. 273, 291 (2004).

[7] X. H. Zhang, N. Maeda, and V. S. J. Craig, Langmuir 22, 5025 (2006).

[8] B. M. Borkent, S. S. de Beer, F. Mugele, and D. Lohse, Langmuir 26, 260 (2010).

[9] L. Zhang, Y. Zhang, X. Zhang, Z. Li, G. Shen, M. Ye, C. Fan, H. Fang, and J. Hu, Langmuir 22, 8109 (2006).
[10] X. H. Zhang, G. Li, N. Maeda, and J. Hu, Langmuir 22, 9238 (2006).

[11] S. Yang, S. M. Dammer, N. Bremond, H. J. W. Zandvliet, E. S. Kooij, and D. Lohse, Langmuir 23, 7072 (2007).

[12] B. M. Borkent, S. M. Dammer, H. Schonherr, G. J. Vancso, and D. Lohse, Phys. Rev. Lett. 98, 204502 (2007).

[13] X. H. Zhang, A. Khan, and W. A. Ducker, Phys. Rev. Lett. 98, 136101 (2007).

[14] S. Yang, E. S. Kooij, B. Poelsema, D. Lohse, and H. J. W. Zandvliet, Euro. Phys. Lett. 81, 64006 (2008).

[15] X. H. Zhang, A. Quinn, and W. A. Ducker, Langmuir 24, 4756 (2008).

[16] S. Yang, P. Tsai, E. S. Kooij, A. Prosperetti, H. J. W. Zandvliet, and D. Lohse, Langmuir 25, 1466 (2009).

[17] M. A. Hampton and N. V. Nguyen, Adv. Colloid Interface Sci. 154, 30 (2010).

[18] V. S. J. Craig, Soft Matter, doi:10.1039/c0sm00558d (2010). 
[19] S. Das, J. H. Snoeijer, and D. Lohse, Phys. Rev. E 82, 056310 (2010).

[20] W. A. Ducker, Langmuir 25, 8907 (2009).

[21] M. P. Brenner and D. Lohse, Phys. Rev. Lett. 101, 214505 (2008).

[22] N. Kameda and S. Nakabayashi, J. Colloid Interface Sci. 461, 122 (2008).

[23] S. M. Dammer and D. Lohse, Phys. Rev. Lett. 96, 206101 (2006).

[24] C. Fradin et al., Nature 403, 871 (2000).

[25] S. Mora, J. Daillant, K. Mecke, D. Luzet, A. Braslau, M. Alba, and B. Struth, Phys. Rev. Lett. 90, 216101 (2003).

[26] F. Jin, J. Li, X. Ye, and C. Wu, J. Phys. Chem. B 111, 11745 (2007).

[27] V. B. Fainerman, E. H. Lucassen-Reynders, and R. Miller, Colloids Surf., A 143, 141 (1998).

[28] J. A. V. Butler, Proc. R. Soc. Ser. A 138, 348 (1932).

[29] S. I. Karakashev, A. V. Nguyen, and J. D. Miller, Adv. Polym. Sci. 218, 25 (2008).

[30] E. H. Lucassen-Reynders, Colloids Surf., A 91, 79 (1994).

[31] I. Prigogine, The Molecular Theory of Solutions (North-Holland, Amsterdam, 1968).

[32] E. A. Guggenheim, Mixtures (Clarendon, Oxford, 1952).

[33] R. C. Read, J. M. Prausnitz, and T. K. Sherwood, The Properties of Gases and Liquids, 3rd ed. (McGraw-Hill, New York, 1977).
[34] S. I. Karakashev and E. D. Manev, J. Colloid Interface Sci. 248, 477 (2002).

[35] S. I. Karakashev, R. Tsekov, and E. D. Manev, Langmuir 17, 5403 (2001).

[36] H. Diamant and D. Andelman, J. Chem. Phys. 100, 13732 (1996).

[37] V. B. Fainerman, Zh. Fiz. Khim. 56, 2506 (1982).

[38] E. D. Manev, S. V. Sazdanova, R. Tsekov, S. I. Karakashev, and A. V. Nguyen, Colloids Surf. A 319, 29 (2008).

[39] V. B. Fainerman and E. H. Lucassen-Reynders, Adv. Colloid Interface Sci. 96, 295 (2002).

[40] R. Wuestneck, R. Miller, J. Kriwanek, and H.-R. Holzbauer, Langmuir 10, 3738 (1994).

[41] V. B. Fainerman, R. Miller, R. Wuestneck, and A. V. Makievski, J. Phys. Chem. 100, 7669 (1995).

[42] M. Ueno, Y. Takasawa, H. Miyashige, Y. Tabata, and K. Meguro, Colloid Polym. Sci. 259, 761 (1981).

[43] J. R. Li, A. Marocco, T. J. Su, R. K. Thomas, and J. Penfold, J. Colloid Interface Sci. 158, 303 (1993).

[44] G. A. Smith, S. D. Christian, E. E. Tucker, and J. F. Scamehorn, Langmuir 3, 598 (1987).

[45] A. Berthod and S. Carda-Broch, J. Chromatogr., A 1037, 3 (2004).

[46] Z. Liu, A. M. Jacobson, and R. G. Luthy, Appl. Environ. Microbiol. 61, 145 (1995). 